\title{
ADJUNCTIONS: CATEGORY THEORY \\ VIA NATURAL COMMUNICATION
}


The development of category theory is a natural and perhaps inevitable aspect of the late 20th century emphasis on the conceptual clarification of what specifies and characterizes an object of a mathematical inquiry pertaining to a universe of discourse as a hypostatic entity. The major precursor of this development can be located in the fields of algebraic topology and abstract algebra, where the specification of complex spaces was not based at all on their set-theoretic point-element constitution, but rather on certain algebraic symbolic groups incorporating some invariant characteristic in relation to this complex space.

This articulated a major change in the way of conceiving the conceptual form and function of mathematical objects, which departed from the axiomatic set-theoretic reductionist approach of analysis of objects in terms of pre-determined, or a priori distinguishable elements endowed with some particular externally imposed structure. The emphasis now has been put on the specification of objects in terms of the communicative relations they bear with other objects of the same species, where the notion of a species of structure is now derived from the whole homomorphic constitution of a certain mathematical universe of discourse, conceptualized in turn, by means of the notion of a category that respects or preserves this structural species.

In this respect, the central focus of the categorical way of rethinking the notion of a mathematical object algebraically and structurally can be described as a major transition in the conception and interpretation of what actually characterizes algebraic symbolic structures. More precisely, it represents a transition from a substantial to a hypostatic concept of structure. In the substantial constitutional settheoretic mode of thinking, structures of any conceivable morphe are defined on a set-theoretic foundational basis, as sets of elements endowed with appropriate structural relations, like the ones characterizing a group structure.

In the hypostatic communicative category-theoretic mode, the emphasis is placed on the kind of homomorphisms among the objects of a category devised to capture and preserve a certain structure as its instances, by means of the pertinent structural constraints on these homomorphisms. In this sense, the notion of structure does not refer substantially to a fixed universe of sets of predetermined elements, but hypostatically acquires a variable reference to other generalized universes, called topoi.

In particular, the hypostatic structural elaboration of an object by way of its variable reference to a topos, to be thought of as a scaffolding entailed contextually by communication, and not as a foundation necessitated absolutely by constitutional needs, points to an indirect 
"obstacle-oriented" approach to the specification of an object as an algebraic structure arising from the category to which it pertains in as an instance. Most important, the specification of objects of a structural species can be even enunciated in terms of the heteromorphic relations

they bear with objects of another structural species, which are thought of as partial resolving probes or covers of the former, under the proviso that these relations can be appropriately internalized within the former category via adjunctions.

The basic categorical principles can be expressed concisely as follows:

i To each kind of mathematical symbolic structure, there corresponds a category whose objects have that structure, and whose homomorphisms preserve it.

ii To any natural morphism on structures of one kind, yielding structures of another kind, there corresponds a functor from the category of the first kind to the category of the second. The implementation of this principle is associated with the fact that a morphism is not merely a function from objects of one kind to objects of another, but must preserve the essential structural relationships among objects.

iii To each natural translation between two functors having identical categorical domains and codomains there corresponds a natural transfiguration, called a natural transformation between these functors that can be restricted to a natural isomorphism of functors.

iv To any canonical bidirectional functorial correlation between two kinds of mathematical structures there corresponds an adjunction, expressed by a pair of adjoint functors between the corresponding categories. An adjunction is equivalent to a categorical process of metaphora effecting the natural communication between the correlated hypostatic structural kinds.

Therefore, if the standard framework of mathematical category theory is to be thought of as a conceptual pyramid based on the notion of a category and converging at the top on the notion of a categorical adjunction, up through the intermediate layers of functors and natural transformations, the emphasis on natural communication by contrast requires a conceptual inversion of this form. Precisely speaking, it is the need for expressing the conceptual norms of natural communication between two different hypostatic levels - the norms of the metaphora between two different hypostases conceived algebraically and structurally from a non-absolute elemental standpoint - that requires the notions of natural transformations and functors, and ultimately the notion of a category. 
As we shall discuss later on, the inverted pyramid of category theory, emphasizing the natural communication between two different hypostatic structural kinds, is intimately related to an "obstacle-oriented" approach to the notion of what a categorical object is. More concretely, the enunciation of the categorical structure of an object of an unknown, novel, or even not directly accessible species, requires a potential cyclically-embracing process of metaphora through another comprehensible categorical species, together with the canonics of this heteromorphic metaphora, so illuminating the former structure by natural communication, or equivalently, descending to and ascending from another covering or resolving structure.

We outline below the standard basic definitions to be found in this order in standard formalist presentations of category theory, and further on, we are going to show how they actually arise inversely via the canonics and norms characterizing the natural-communication of non-absolute hypostatic structural kinds.

CATEGORIES:

A category $\mathcal{C}$ is a class of objects and morphisms of objects such that the following properties are satisfied:

i For any objects $X, Y$ all morphisms $f: X \rightarrow Y$ form a set denoted $\operatorname{Hom}_{\mathcal{C}}(X, Y)$;

ii For any object $X$ an element $i d_{X} \in \operatorname{Hom}_{\mathcal{C}}(X, X)$ is distinguished; it is called the identity morphism;

iii For arbitrary objects $X, Y, Z$ the set mapping is defined

$$
\operatorname{Hom}_{\mathcal{C}}(X, Y) \times \operatorname{Hom}_{\mathcal{C}}(Y, Z) \rightarrow \operatorname{Hom}_{\mathcal{C}}(X, Z)
$$

For morphisms $g \in H_{\mathrm{Com}}(X, Y), h \in \mathrm{Hom}_{\mathcal{C}}(Y, Z)$ the image of the pair $(g, h)$ is called the composition; it is denoted $h \circ g$. The composition operation is associative.

For any $f \in \operatorname{Hom}_{\mathcal{C}}(X, Y)$ we have $i d_{Y} \circ f=f \circ i d_{X}=f$.

For an arbitrary category $\mathcal{C}$ the opposite category $\mathcal{C}^{o p}$ is defined in the following way: the objects are the same, but $\operatorname{Hom}_{\mathcal{C}^{o p}}(X, Y)=\operatorname{Hom}_{\mathcal{C}}(Y, X)$, namely all arrows are inverted. A category $\mathcal{C}$ is called small if the classes of its objects and morphisms form genuine sets respectively. 
FUNCTORS:

Let $\mathcal{C}, \mathcal{D}$ be categories; a covariant functor $\mathbf{F}: \mathcal{C} \rightarrow \mathcal{D}$ is a class mapping that transforms objects to objects and morphisms to morphisms preserving compositions and identity morphisms:

$$
\mathbf{F}\left(i d_{X}\right)=i d_{\mathbf{F}(X)} ; \mathbf{F}(g \circ f)=\mathbf{F}(g) \circ \mathbf{F}(f)
$$

A contravariant functor $\hat{\mathbf{F}}: \mathcal{C} \rightarrow \mathcal{D}$ is, by definition, a covariant functor $\mathbf{F}: \mathcal{C}^{o p} \rightarrow \mathcal{D}$.

\section{NATURAL TRANSFORMATIONS:}

Let $\mathcal{C}, \mathcal{D}$ be categories, and let further $\mathbf{F}, \mathbf{G}$, be functors from the category $\mathcal{C}$ to the category $\mathcal{D}$. A natural transformation $\tau$ from $\mathbf{F}$ to $\mathbf{G}$ is a mapping assigning to each object $A$ in $\mathcal{C}$ a morphism $\tau_{A}$ from $\mathbf{F}(A)$ to $\mathbf{G}(A)$ in $\mathcal{D}$, such that for every arrow $f: A \rightarrow B$ in $\mathcal{C}$ the following diagram in $\mathcal{D}$ commutes;

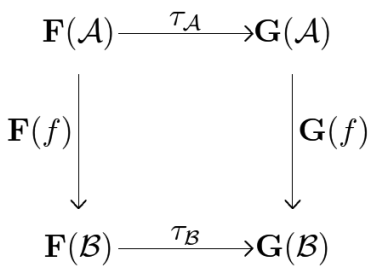

That is, for every arrow $f: A \rightarrow B$ in $\mathcal{C}$ we have:

$$
\mathbf{G}(f) \circ \tau_{A}=\tau_{B} \circ \mathbf{F}(f)
$$

\section{NATURAL ISOMORPHISMS:}

A natural transformation $\tau: \mathbf{F} \rightarrow \mathbf{G}$ is called a natural isomorphism if every component $\tau_{A}$ is invertible.

ADJOINT FUNCTORS:

Let $\mathbf{F}: \mathcal{C} \rightarrow \mathcal{D}$ and $\mathbf{G}: \mathcal{D} \rightarrow \mathcal{C}$ be functors. We say that $\mathbf{F}$ is left adjoint to $\mathbf{G}$ (and correspondingly that $\mathbf{G}$ is right adjoint to $\mathbf{F}$ ), if there exists a bijective correspondence between the arrows $\mathbf{F}(C) \rightarrow D$ in $\mathcal{D}$ and $C \rightarrow \mathbf{G}(D)$ in $\mathcal{C}$, which is natural in both $C$ and $D$.

$$
\mathbf{F}: \mathcal{C} \rightleftarrows \mathcal{D}: \mathbf{G}
$$


Pictorially we have;

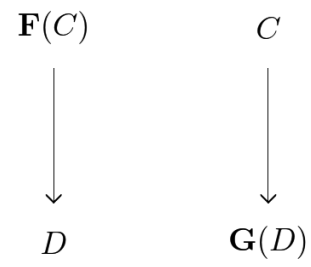

where the left part is in $\mathcal{D}$ and the right in $\mathcal{C}$. Then, we say that the above pair of adjoint functors constitute a categorical adjunction.

DIAGRAMS:

A diagram $\mathbf{X}=\left(\left\{X_{i}\right\}_{i \in I},\left\{F_{i j}\right\}_{i, j \in I}\right)$ in a category $\mathcal{C}$ is defined as an indexed family of objects $\left\{X_{i}\right\}_{i \in I}$ and a family of morphisms sets $\left\{F_{i j}\right\}_{i, j \in I} \subseteq \operatorname{Hom}_{\mathcal{C}}\left(X_{i}, X_{j}\right)$.

COCONES:

A cocone of the diagram $\mathbf{X}=\left(\left\{X_{i}\right\}_{i \in I},\left\{F_{i j}\right\}_{i, j \in I}\right)$ in a category $\mathcal{C}$, consists of an object $X$ in $\mathcal{C}$, and for every $i \in I$, a morphism $f_{i}: X_{i} \rightarrow X$, such that $f_{i}=f_{j} \circ f_{i j}$ for all $j \in I$, that is, such that for every $i, j \in I$, and for every $f_{i j} \in F_{i j}$ the diagram below commutes

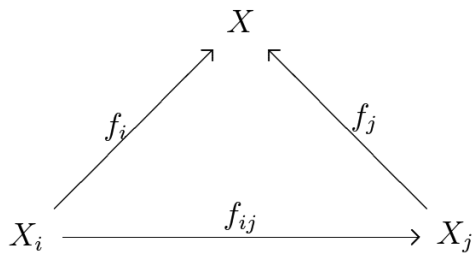

COLIMITS:

A colimit of the diagram $\mathbf{X}=\left(\left\{X_{i}\right\}_{i \in I},\left\{F_{i j}\right\}_{i, j \in I}\right)$ is a cocone with the property that for every other cocone given by morphisms $f_{i^{\prime}}: X_{i} \rightarrow X^{\prime}$, there exists exactly one morphism $f: X \rightarrow X^{\prime}$, such that $f_{i^{\prime}}=f \circ f_{i}$, for all $i \in I$ (universality property). 
Reversing the arrows in the above definitions of cocone and colimit of a diagram $\mathbf{X}=\left(\left\{X_{i}\right\}_{i \in I},\left\{F_{i j}\right\}_{i, j \in I}\right)$ in a category $\mathcal{C}$, results in the dual notions called cone and limit of $\mathbf{X}$ respectively. Moreover, starting with a diagram $\mathbf{X}=\left(\left\{X_{i}\right\}_{i \in I},\left\{F_{i j}\right\}_{i, j \in I}\right)$ in a category $\mathcal{C}$, that consists only of the objects $X_{i}, i \in I$, as nodes but without morphisms, that is all $F_{i j}=\varnothing$, we obtain the notion of the categorical coproduct, $\coprod_{i \in I} X_{i}$ (as a special colimit) and product, $\prod_{i \in I} X_{i}$ (as a special limit) respectively. The morphisms $f_{i}$ in the corresponding definitions are called canonical injections of the coproduct and canonical projections of the product, respectively. We emphasize that we can derive special notions of limits and colimits, corresponding to the shape of the base diagram $\mathbf{X}$. In this sense we obtain the following; an initial object is the colimit of the diagram consisting of the empty set. A coequalizer is the colimit of a diagram consisting of two parallel arrows $A \Rightarrow B$. A pushout is the colimit of a diagram of the form:

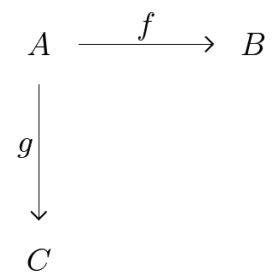

The dual notions are the following: a terminal object is the limit of the diagram consisting of the empty set. An equalizer is the limit of a diagram consisting of two parallel arrows $A \Rightarrow B$. A pullback is the limit of a diagram of the form:

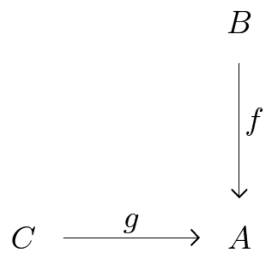


The natural communication between two different hypostatic structural kinds, conceptualized as different categories, is inextricably tied to an "obstacle-oriented" approach to the notion of what a categorical object is. It implies that the categorical structure of an object of a non-directly comprehensible or novel structural species, requires a potential cyclicallyembracing process of metaphora through another categorical species. Since these species occupy different hypostatic structural levels the metaphora is inevitably of a heteromorphic nature. This means that a certain structural canonics is required for the accomplishment of the metaphora. The basic idea is that the metaphora bears the potential to illuminate the unknown or obstacle-laden structure by heteromorhically descending to and ascending from another comprehensible categorical hypostatic level.

The effectiveness of this metaphora is based on two factors: First, the directly comprehensible level should consist of categorical objects whose hypostasis should be somehow illuminating in relation to the ones of the obstacle-laden level. In this sense, ideally these categorical objects should manifest some local or partial structural spectral invariance characterizing the objects of the former species. In other words, their suitability should be based on their capacity to encode some local or partial structural invariant which is crucial for disclosing the structure of the former ones. It is precisely in this manner that they can be considered as structurally illuminating in terms of their categorical hypostasis. Secondly, since the metaphora is heteromorphic, a certain canonics must be in force that allows the natural communication between these levels. In other words, the objects of the comprehensible kind should be adjoined as probes or generalized pointers in the environment of the former ones not in a rigid, but in a plastic manner, so that connectively, and not only collectively, they can enforce a homeotic cobounding relation to an object of the unknown species that has the capacity to play the role of a structural canon for this object.

In turn, this qualification serves to facilitate the inverse canonization of the employed probes as icons for the deployment of the $a b$ initio unknown object at least within the symbolic algebraic milieu. Note that in this categorical context of thinking the notion of an icon is abstracted from its direct visual connotation and is elevated at the symbolic algebraic level as a local or partial invariant means of internal illumination through its canonization. Therefore, the symbolic and structural specification of the unknown via metaphora through the connective network of icons cobounding it structurally serves to establish the structural adaptability of the icons to the species of the unknown as its partially covering spectral structural invariants. In this sense, the 
unknown object is illuminated connectively by this network of icons, while the latter are canonized reciprocally by their participation in the canon, which works metaphorically to unfold the structure of the former. Finally, and in terms of this canonization procedure, a gnomon emerges at the categorical level of the unknown structural species by the adjunction of the categorical level of its icons.

It is precisely these factors that we maintain fundamental for a careful re-evaluation of the novel mode of object-specification induced by the development of category theory in mathematical thinking in relation to the objective of natural communication. More precisely, the notion of a structural symbolic icon is conceived as the principal means of probing or resolving an object of an obstacle-laden species in a canonical manner, independently of any a priori requirement of analysis of this object in its set-theoretic elements. This is the case because the notion of a canonized icon is not subordinate to any analytic reduction, but on the contrary, subsumes a well-defined structural characterization derived from its canonical internalization within the category deciphering the species of the object under investigation. In other words, a probe is qualified as an icon if and only if it can be canonized and thus internalized within the category in question. Technically, this simply means that it can give rise to a structure-respecting homomorphism within this category targeting the object of inquiry. An immediate consequence of this characterization is that a canonized action is structurally adaptable by means of adjunction to the species of the investigated object.

The major issue arising in this state of affairs is the detection of the conditions that cast a probe into a canonized icon; these are the very conditions, conditions that render a probe canonically structurally adaptable to the object of inquiry, so that it can function as a source of local or partial spectral illumination on this object. Intuitively, since a probe should furnish a frame for resolving the unity of the investigated object, this frame can be structurally adapted to the species of the object, if and only if it encodes some structural invariant feature pertaining to the level of resolution or distinguishability of the investigated object with respect to the frame employed. The requirement of structural adaptability qualifies a probe as an illuminating symbolic icon, which plays the role of a local or partial structural frame for resolving or partitioning the investigated object under the action of this probe by virtue of the structurally invariant context it discloses. Concomitantly, the action of a canonized icon becomes co-extensive to a local or partial cover of the object of enquiry subordinate to the aphairetic filtering, or sieving, spectral invariant capacity of the icon. The covering action leads to a localization of the object of enquiry, thereby, it also gives rise to a topology on the object of enquiry only under the mild constraints of transitivity and compatibility of the covers under the operation of restriction to subcovers. 
It has to be stressed that the implicated notion of localization with respect to an icon is derived internally and intrinsically only from the specific aphairetic structural invariant capacity of this icon in its function as a local cover, and not from any spatial embedding environment of any external kind. In this respect, an icon, although incomplete in its capacity to illuminate and resolve the investigated object globally or in its entirety, shapes this object locally or partially in a structurally adaptable manner. As a result, it can be inversely, internally extended beyond its compatible restriction as well, under the proviso that overlaps compatibly with some other icon deciphering another local cover of the investigated object.

A crucial feature of this local iconic schematism of an object in a category is that it does not assume or require the existence of an allencompassing icon, meaning that any single particular icon should not be thought of as an independent part of an all-encompassing icon. In contradistinction, the iconic schematism of an object is based on the idea of a multiplicity of partially illuminating icons, covering the object connectively only under their joint canonic cobounding action. It is equally vital to point out that such a jointly covering multiplicity of icons is not merely a set of icons. It may be thought of as a colimiting, and hence, spectrally cobounding object, comprehending within itself the joint resolving spectral capacity of all contributing icons, participating in its integrity, coherently and compatibly synthesized in a way which does not require the treatment of those icons as independent parts.

Rather it may be visualized as a multi-layered granulation weaving sieve, whose variable concatenated openings comprise the resolving or illuminating power of the corresponding icon, and which becomes structurally adaptable to the categorial species of the investigated object. This colimiting, or cobounding, object arises out of heteromorphic canonics, by means of a categorical adjunction, in the sense that the canon pertaining to the joint spectral capacity of all the partially illuminating icons induces a gnomon for the object of enquiry under homomorphic internalization within its categorical species of structure.

In light of the above, it becomes possible to appreciate the significance of the central formal theorem of category theory - the Yoneda-Grothendieck lemma - for the proposed obstacle-oriented approach, referring to the means of articulability of an object in a category. More precisely, this theorem states formally that an object is completely specified by the network of all morphisms directed to this object by all other objects in the same category. In other words, an object in a category can be completely resolved, or partitioned, or even classified and retrieved, and thus become totally illuminated, by all internalized structure-respecting arrow-morphisms pointing towards it within the same category. The complete illumination promised by this theorem rests on the fact that the whole network of pointing morphisms specifies, and thus articulates, the object uniquely up to canonical isomorphism. Thus, 
it provides the universal means of articulation of the object in relation to its categorical structural species, in the sense that its specification as outlined is unique up to equivalence, established by an explicitly demonstrable isomorphism.

The ground-breaking consequence of this theorem is essentially that the object of interest can be legitimately subjugated or even conceptually substituted by the network of all internalized structurerespecting relations or morphisms targeting it within the same category. For this reason, the object constitutes a formal symbolic representation of the whole network of relations directed to it within its categorial species, and inversely, this network becomes uniquely representable symbolically up to equivalence by the targeted object. Note that the term "representation" is used in the formal sense, meaning that the object becomes the symbolic placeholder of the network of morphisms pointing to it. We would like to enhance this non-spatial notion of representation with a temporal connotation that emphasizes its synthetic functional role in this setting. In particular, representation concerns the depth of the present in its capacity to instantiate an object by encapsulating all the morphism pointing to it. In other words, the synthetic act of representability in category theory is enunciated temporally by means of the capacity of an instantiated object to encapsulate all possible paths of arrows pointing toward it, which in turn, constitute its indexical iconization. From now on, this is precisely the meaning that we attribute to the technical term "representation" in the context of category theory.

In practice, the specification of an object in a category by the network of all possible morphisms directed to it within the same category is redundant. It is precisely here that the notion of an internalized structurally adaptable, and thus structure-respecting, probing relation, emanating from another categorical species of structure, becomes significant. The underlying idea is that subject to theoretical, experimental, or computational reasons, a category of probes is always delineated in relation to an investigated object of some unknown, nondirectly accessible, or obstacle-laden categorial species. This category of probes may have the status of a subcategory of the category in which the investigated object is structurally placed, but this is no general requirement. What is crucial, is that the action of a probe can be structurally adapted as an internalized, and therefore, structurerespecting directed morphism within the category of the investigated object. Henceforth, it is exactly this qualification of a probing relation that gives rise to the notion of an icon illuminating an investigated object of some category.

The natural issue arising in this setting is to leverage the redundancy in the specification of an object in a category through the network of all possible relations directed to it, by restricting this network to a minimal but sufficient assemblage of icons capable of collectively and 
connectively illuminating this object. Intuitively, the sufficiency condition pertains to the joint covering action of the object by the utilized assemblage of icons, as we have already seen. Under this restriction of the classifying network of internalized structure-respecting, directed morphisms on the investigated object in iconic schematic terms, the validity of the category-theoretic object-specification theorem remains intact. Namely, an object of a categorical structural species can be specified, classified, and retrieved by the assemblage of all partially illuminating icons upon it, uniquely up to equivalence. The objective is that this assemblage can be appropriately qualified as a heteromorphic canon capable of establishing natural communication between the categorical species of the object of enquiry and the species of the icons. The underlying idea again is that the heteromorphic canon naturally induces a gnomon for the investigated object under homomorphic internalization in its categorical species of structure.

An immediate consequence of the above is that for a fixed object under investigation, the network or assemblage of all icons directed towards it can be objectified category-theoretically into a novel object of a higher structural species, i.e. an iconic schematism functor, called simply a representable functor. The root of this terminology is that an iconic schematism functor becomes symbolically representable by the investigated object up to equivalence. An iconic schematism functor is a structure-respecting morphism from the category of the investigated object into the category of sets. That is to say, for each internalized source-probe it assigns a set of icons capable of partially illuminating the unknown object from the specified source. Clearly, the iconic schematism functor of the investigated object varies over all possible internalized source-probes and is also evaluated at each one of them by producing the set of icons corresponding to it. Then, the meaning of the previously expressed object-specification theorem boils down to the fact that the categorical species of the investigated object can be fully and faithfully embedded into the category of functors from this species to sets, where the category of sets is used as a scaffolding for this purpose. Henceforth, it can be identified by means of the representable functors, i.e. the respective iconic schematism functors, technically symbolized as Homfunctors. It is instructive again to think of an iconic schematism functor, representable by the investigated object up to equivalence, as a multilayered granulation sieve whose variable concatenated openings comprise the resolving or illuminating power of the corresponding icons.

The heteromorphic natural communication emerges in categorical terms from the conceptual inversion of an iconic schematism functor. In general, if an iconic schematism functor is considered as an encoding functor of the unknown categorical species to the species of its icons, then its inversion would be a decoding functor in the opposite direction. Pairs of encoding/decoding functors give rise to categorical adjunctions, where 
these functors are called adjoint to each other. The notion of an adjunction as an expression of heteromorphic natural communication constitutes a far-reaching generalization of the algebraic notion of an equation. Precisely, the iconic schematism functor furnishes the algebraic variables of this equation with respect to the scaffolding of the category of sets, whereas its conceptual inverse decoding functor amounts to the solution of this equation, i.e. the specification of these variables.

More concretely, we have already concluded that an investigated object of some categorial species can be conceptually substituted due to the amenability of its classifying iconic schematism functor to symbolic representation by the former up to equivalence. The important point is that the iconic schematism functor and the representing object of this functor, are located within different categorical species of structure. Thus, the pertinent issue again is how the iconic schematism functor, or equivalently, a whole sieve of icons directed towards an object of inquiry, becomes structurally adaptable as a totality to the categorial species of this object. The structural adaptability of the iconic schematism functor, and thus, the internalization of the whole sieve of icons within the categorial species of the investigated object, is possible only as a colimiting canon pertaining to the joint spectral capacity of all the partially illuminating icons.

The pronounced inversion consists in the realization that the iconic schematism functor on an object of inquiry, after the selection of a certain category of probes - as structurally invariant iconic contexts of resolving and illuminating this object at various layers of distinguishability - is sufficient to depict, approximate, or even completely disclose the categorical species of the investigated object, up to equivalence. Note that in this setting, the categorical structural species of the investigated object, and not merely the investigated object itself, is considered an unknown variable. Thus, the proposed conceptual inversion can be utilized as a recognition principle of a new categorial species of structure. This becomes possible after the initial selection of a category of probes applied to a hypothetical object of a species unknowable a priori, which gives rise - modulo all the previously-stated requirements - to the iconic schematism functor of this object of inquiry.

This constitutes a novel approach to revealing new categorial species of structure by means of heteromorphic natural communication, through the employment of an encoding iconic schematism functor defined in terms of structurally invariant contexts for resolution of this unknown species, and then inverting or decoding it back. We may schematically assert that the categorical recognition principle formulated in terms of natural communication turns the formal object-specification theorem of category theory onto its head, in the sense that, considering all practical or theoretical applications, it is the categorical species of the investigated object that is the real unknown and not the object itself. 
Thus, we finally arrive at the crucial point of formulating the proclaimed recognition principle of some new categorical species of structure given the set-up of a representable functor, i.e. of an iconic schematism functor, as previously. A posteriori, this constitutes an interpretation of the category-theoretic syntax from the standpoint of natural communication. More tellingly, we read from Saunders MacLane, one of the two inventors of the categorical syntax, the following cryptic remark: "But I emphasize that the notions category and functor were not formulated or put in print until the idea of a natural transformation was also at hand".

This is at odds with the usual axiomatic presentation of the subject, more accurately to be read as its inversion. Of course, for reasons of systematic presentation of the category-theoretic syntax, the definition of a natural transformation, being a morphism of functors, has to be preceded by the definitions of what a functor is and what a category is. Notwithstanding this fact, the idea of a natural transformation is solely based on the concept of covariance, meaning that the associated morphism of functors to which it refers, is not subordinate to any ad hoc choice of underlying object to which these functors can be applied and evaluated. In other words, a natural transformation between two functors pertains to the categorial species of the underlying objects and not to the objects themselves. This is the decisive abstraction for the formulation of the recognition principle of obstacle-laden categorical species of structure in natural communication terms.

Up to present, we have reached the conclusion that the recognition principle of some new categorical species of structure, should be properly expressed by means of a natural transformation of functors. It remains to examine the particularities of this natural transformation in detail, i.e. which precise morphism of functors is pertinent for the formulation of the recognition principle. What we have at our disposal is the set-up of an iconic schematism functor for each unknown hypothetical object of this new categorial species, after the selection of a category of probes, namely a category of structurally invariant contexts for resolution of these objects in terms of icons, according to the preceding argument. What we have also established is that given an iconic schematism functor on an object of inquiry, the associated sieve of all icons on this object becomes internalized as a totality in its categorial species by means of a novel colimiting or cobounding object, pertaining to the joint connective resolution capacity of all the involved icons illuminating this object. It is significant that this internalization process by means of a colimiting object is universal, in the sense that it is uniquely specified up to equivalence; it is unique up to a canonical isomorphism. In the terms of categorical natural communication, the universality property gives rise to a heteromorphic canon, which finally induces a homomorphic 
gnomon for the investigated object under internalization in its categorical species of structure.

Therefore, a colimiting object uniquely determines the universal terminus through which each one of the icons should factor in its function to probe and illuminate the species of the object of enquiry, being in this sense its gnomon. Clearly, the instantiation of such a colimiting object pertains to any iconic schematism functor associated with any object of the unknown categorial species. This prompts the realization that the iconic colimiting or cobounding process is actually a functor from the category of iconic schematism functors to the sought-after novel category. Most important, this colimiting functor conceptually inverts the iconic schematism functor on each object of the unknown species, because of the universality property characterizing the determination of the colimit object with respect to each object of the unknown species.

Put equivalently, for each object of the unknown species the set of all its partially illuminating icons factors uniquely through the colimiting object of the corresponding iconic schematism functor pointing to it. Henceforth, any object of the unknown species can be recognized uniformly and universally only through such a corresponding colimiting object terminus of an iconic schematism functor on it. In more philosophical terms, this means that the colimiting functor actually determines the genus of the whole new categorical species of structure in relation to the underlying category of icons, those structurally invariant contexts for resolution or illumination of the objects of this new species. Note that the notion of genus incorporates the recognition principle of the whole new categorical species of structure and not only of the objects of this species.

In a nutshell, for each object of the unknown categorical species of structure, after setting up an iconic schematism functor referring to it, we compute and apply the colimiting functor on the latter, thus, conceptually inverting it. This process is sufficient to determine the cobounding terminus uniquely up to equivalence, and thus, the universal homomorphic gnomon pertaining to the new categorical species through which the heteromorphic canonics of any icon has to factor in order to illuminate the object of inquiry. Since the same process applies uniformly and universally to any object of the unknown species, it is elevated to a process of articulation of the genus of the whole new categorical species. Henceforth, the unknown or obstacle-laden categorical species is recognized in iconic spectral terms by means of its genus determination through natural communication, where the iconic schematism and the colimiting functors play the role of the encoding and decoding bridges respectively.

We are now ready to express the recognition principle of some new categorical species of structure through natural communication, by means of a natural transformation of functors that has been our main 
objective. Conceptually, the leading idea is that object specification of an unknown categorical species in iconic terms always follows from genus determination of these species as a whole, as above. The unknown categorical species can equivalently be thought of, and objectified functorially, as the identity functor on itself. In this light, the recognition principle of this categorial species as a whole -irrespectively of any ad hoc choice of objects - should be expressed as a natural transformation of the identity functor induced by any appropriate selection of a category of probes to function as icons in relation to this categorical species. The suitability of a category of probes, furnishing structurally invariant contexts for resolving any object of the unknown species in iconic terms, is judged from its capacity to induce genus determination of this categorial species. This is possible if and only if a heteromorphic canon of natural communication is activated, which in turn, instantiates a universally cobounding gnomon of icons as a terminus of spectral recognition for each object of the sought-after species in a uniform manner. Equivalently, the heteromorphic canon is functorially tenable, if and only a colimiting functor exists whose action on the iconic schematism functor results in a natural transformation of the identity functor. Under these conditions, we say that the unknown categorical species is recognized by means of a natural communication monad, in the sense that the unveiling of these species emanates from the joint and interlinked illuminating capacity of its probes-icons.

Consequently, the recognition principle of some new categorical species of structure, initially taken to be unknown, is expressed as a natural transformation of the identity functor on this species by the endofunctor acting on the same species, which is obtained by composing the colimiting functor with the iconic schematism functor, depending on the appropriate selection of an underlying category of probes to act as icons. This natural transformation of the identity functor, determines the genus of the new categorical species in iconic terms, thereby establishing the sought-after recognition principle of this species. It is worth remarking that the above recognition-inducing natural transformation of the identity functor is equivalent to the conceptual inversion of the iconic schematism functor. More precisely, in technical terms, the colimiting functor that inverts the iconic schematism functor is called a left adjoint to the latter, since it acts on the left of it so that their composition provides the determining endofunctor on the unknown categorial species. It is precisely this determining endofunctor that plays the role of a heteromorphic canon of natural communication; it gives rise to a communicability monad between the species of the icons and the obstacle-laden species, which bears the capacity to unveil the latter in terms of the former.

We note that the notion of adjoint functors was first conceived, abstracted, and formulated more than a decade after the introduction of 
the category-theoretic framework in terms of categories, functors and natural transformations. Before the introduction of this notion category theory can be viewed as a useful tool for organizing, systematizing and classifying various species of already known mathematical objects. In other words, it served more as a theory of taxonomy than as a theory of invention and discovery. The notion of adjoint functors transformed its role completely and paved the way for forming bridges between seemingly unrelated mathematical disciplines; in short, it revealed its natural communication underpinnings. It is precisely this notion that discloses the philosophical significance of category theory, and at the same time, makes it suitable for solving difficult structural problems in the natural sciences.

The formulation of the theory of adjoint functors in categorytheoretic terms has its roots in homology theory and algebraic topology. We arrived at this notion by following another more philosophical route involving the objective of heteromorphic natural communication, if expressed in terms of the category-theoretic syntax. Since the canonics characterizing this kind of natural communication, pertaining to categorical species of structure, is not to be taken for a merely taxonomic tool, but as a potent instrument of genuine emergent novelty, the basic problem posed is the means of recognition of an obstacle-laden categorical species in partial or local iconic terms. As we showed, this involves first, the setting-up of an iconic schematism functor, after the selection of an underlying category of probes in their function as icons, i.e. partially or locally congruent structural invariant contexts for illuminating and connectively resolving an object of the sought-after categorial species. The solution to this problem, which a posteriori characterizes the suitability of the selected category of probes and the sufficiency of the icons, requires the conceptual inversion of an iconic schematism functor. This conceptual inversion is equal in effect to finding a colimiting functor acting on a corresponding iconic schematism functor as a left adjoint, and thus, inverting it.

In turn, the above inversion embraces and indirectly solves the problem posed by means of the heteromorphic canonics establishing the natural communication between the categorical species of interest, that is, by means of a pair of adjoint functors between these species. In turn, the solution amounts to a natural transformation of the identity functor on the unknown categorical species, which is thus, recognized gnomonically by means of the unique homomorphic termini induced by the colimiting functor for each object of the sought-after species. Accordingly, the conceptual inversion of an iconic schematism functor leads to the genus determination of the initially unknown categorical species by means of the established recognition principle. Under certain conditions, the natural transformation of the identity functor solving the problem of recognition of a new categorical species of structure, can be 
restricted to a natural isomorphism. In this case, each object of this new species is specified uniquely up to equivalence by the corresponding colimiting or cobounding object emerging by the left adjoint to the iconic schematism functor referring to it. These conditions are usually implemented by imposing a particular categorical topology on the underlying category of probes, which amounts to localizing an assemblage of icons into a sheaf of icons for this topology.

The modus-operandus of physics as a natural science is based on observation and measurement. In particular, measurement is the process corresponding to a well-defined observational procedure, according to which, various attributes or magnitudes are assigned numbers, or more generally, "number-like" quantities. In this sense, the general notion of number is understood physically as the outcome of a measurement corresponding to an observational procedure. The abstraction of the measurement process gives rise to the notion of coordinatization or arithmetization. The power of the measurement process to arithmetize phenomena according to a well-defined underlying observational procedure is in essence the objectification of magnitudes, in the sense that they can be communicated to other observers, and thus become amenable to comparison and transformation, again according to prescribed rules. Furthermore, the "number-like" quantities obtained by the coordinatization process can be subjected to algebraic operations, so that they can form suitable algebraic structures closed under the action of the corresponding operations.

Hence, from a physical perspective, algebraic structures of "number-like" quantities of any particular operational form, can be thought of as solutions to a physical measurement problem. Most commonly, the effectuation of the coordinatization process itself requires the conceptual extension of what can function as a "number-like" quantity. In turn, this is reflected in the algebraic process of extension of scalarity, which precisely extends the notion of a "number-like" quantity in a way that preserves the closure requirements under the application of algebraic operations characterizing some structure of already known "numbers". In categorical terms this means that the algebraic process of extension of scalars, solving a physical coordinatization problem, is a functorial process.

We recall that the father of the above described natural philosophy is Thales and his theory of measurement based on proportionality of magnitudes. The sole purpose of Thales' theory of proportions had been the measurement of a not directly accessible magnitude using a gnomon. This refers to the height $x$ of an inaccessible pyramid, given the length $c$ of its accessible shadow, as well as, the height $a$ and shadow length 
$b$ of an accessible object, functioning as a measurement rod. The proportion between magnitudes resolving the Thalesian measurement problem reads as follows:

$a$ to $b$ is as $x$ to $c$

Symbolically, the above proportion is depicted by the equation $\frac{a}{b}=\frac{x}{c}$, from which the not directly accessible magnitude $x$ can be obtained indirectly as $x=a c / b$. We remind that the geometric theory of proportions for the resolution of measurement problems of the Thalesian form, contains the seeds of conception of a group-theoretic structure, together with the concomitant formulation of an algebraic equation for the determination of unknowns.

In this mode of thinking, the geometric resolution of the Thalesian problem, in terms of proportionality, implicitly anticipates the discovery of the multiplicative monoid structure of positive integers, and subsequently, the multiplicative group structure of the rationals and the real numbers. The meaning of this assertion boils down to the realization that the determination of an unknown magnitude in the Thalesian setting, interpreted algebraically, requires the introduction of the multiplicative group structure of the rational or the reals (standing for magnitudes), in order to provide a solution to the associated equation expressing the corresponding proportion. Thus, the determination of unknown magnitudes by the method of proportion, algebraically entails the introduction of the group-theoretic closure structure on magnitudes, equipped with the operation of multiplication and possessing an inverse, which is division.

Up to now, we argued that algebraic structures of "number-like" quantities of any particular operational form, can be thought of as solutions to physical measurability problems. Until recently, these algebraic structures have been conceived as sets endowed with prescribed operations, like addition and multiplication, satisfying a closure condition with respect to the action of these operations on the elements of the underlying sets. Gradually the primary significance of considering the homomorphisms between algebraic structures of the same kind came to collective realization. In particular the existence of an isomorphism between two algebraic structures of the same kind essentially came to mean that these algebraic structures have exactly the same operational role.

Thus, the conception of algebraic structures of some kind should be considered primarily in terms of the relations between them, conceptualized in terms of incoming or outgoing homomorphisms (structure-preserving morphisms), and not in the restricted terms of their elements. The decisive fact in this conceptual re-articulation of algebraic 
structures has been the realization that their elements can be thought of as special homomorphisms from particular algebraic structures of the same kind.

Note that the notion of homomorphism depends on the kind of algebraic structure considered, since it reflects the preservation of the operations between structures of the same kind. Here arises the pure algebraic notion of a "generalized element" of an algebraic structure conceived as an outgoing homomorphism from this algebraic structure into some other of the same kind. Of course, simultaneously the dual notion of a "generalized element" applies, conceived as an incoming homomorphism from some other algebraic structure of the same kind. The artifact of this re-conceptualization requires an interpretational shift in the semantics of structure. More concretely, it is reasonable to coin the term algebraic object of some kind and reserve the structural qualification for the environment where these objects are situated and are related to each other by means of homomorphisms. This is precisely the semantic transition required for the category-theoretic re-interpretation of settheoretic algebraic structures entailed by the primary role of homomorphisms (generalized elements and their duals) in the determination of algebraic objects of some kind.

The definition of a category of algebraic objects of some kind with arrows being homomorphisms between them, constitutes an abstraction of the behavior of functions closed under the associative operation of composition. More precisely, the notion of a function is generalized to the notion of a homomorphism, whereas the associative operation of composition becomes an operation on sets of homomorphisms between algebraic objects of the same kind satisfying the same properties that functions and compositions satisfy.

Note that the composition of two functions $f, g$, denoted as $f \circ g$ is defined only in case that the codomain of $g$ is the domain of $f$. Moreover, the composition of a function $f$ with the identity of either its domain or codomain gives $f$ again. It is interesting then to notice that from a structural set-theoretic viewpoint, a category of algebraic objects of some kind, may be thought of as a partial algebra itself. More precisely, the elements of this algebra are the homomorphisms, and their composition is actually an associative binary operation which is only partial, being defined only when the composition of homomorphisms is meaningful. Then, the role of the objects is just that of labeling the homomorphisms for the determination of the domain of the binary operation of composition.

In a nutshell, the notion of a category of algebraic objects of some kind is a conception based on the behavior of functions closed under the associative operation of composition, abstracted in terms of homomorphisms, which in turn, have been idealized as algebraic 
"generalized elements" (or their duals) completely determining the algebraic objects themselves. Because an obvious duality obtains between incoming and outgoing homomorphisms with respect to an algebraic object, this is built into the definition of a category so that the operation of arrows-reversal leaves the concept of a category invariant, meaning that this operation gives again a category in dual or opposite relation in comparison to the given one.

A consequence of this fact is that all categorical constructions come in dual pairs corresponding to the dual viewpoints from which incoming or outgoing arrows with respect to a constituted algebraic object are seen. Apart from the duality property, we have seen above that the successful effectuation of the coordinatization process in more and more demanding physical measurement problems requires the conceptual extension of the meaning of a "number-like" quantity, described by the algebraic process of extension. For example, the resolution of the Thalesian measurement problem requires the extension of integer numbers into the rational numbers so that division becomes possible. Notably, the algebraic extension process provides algebraic objects of operationally extended "number-like" quantities including the initial ones. Note also that since we should always think in a dual way regarding the direction of arrows with respect to an algebraic object there is clearly an inverse algebraic process called restriction. The semantics of this inverse pair of algebraic processes will be explained in detail subsequently.

Now, the design of the categorical framework of reasoning with respect to the algebraic process of extension provides the conception of the algebraic object of extended "number-like" quantities, of the same kind as the initial one, uniquely up to isomorphism by means of a universal property. More precisely, each outgoing homomorphism $A \rightarrow B$ from the initial object $A$ into a set $B$ endowed with the extra structure, extends to an outgoing homomorphism $A \rightarrow B$ from the algebraic object $A$ newly constructed by extension (solving a corresponding measurement problem) into $B$. Equivalently stated, every homomorphism $A \rightarrow B$ of the previous form uniquely factors through $A$.

\subsection{NATURALITY: FUNCTORS AND COVARIANT TRANSFORMATIONS}

We have seen that the idea of a category of algebraic objects of some kind for which arrows are homomorphisms incorporates from the start the basic idea of duality by arrow reversal, emanating from the fundamental role played by homomorphisms (algebraic "generalized elements") and their dual distinction into incoming and outgoing kinds with respect to an algebraic object completely determined by them. Moreover, understanding algebraic objects as providing solutions to physical 
coordinatization problems, the category-theoretic framework referring to algebraic objects of some kind, forces the conception of operationally extended objects of the same kind, not in terms of their set-theoretic constitution from given elements, but in terms of some universal property determining them up to isomorphism within the same category.

Now, the establishment of the notion of a category of algebraic objects naturally raises the difficulty of defining the notion of a function whose domain and codomain are categories. Obviously such a function should preserve the composition operation binding a category as an associatively closed universe of discourse. This is precisely the notion of functor between categories. From an equivalent viewpoint, since a category may be considered as a partial algebra with respect to the binary operation of composition, the notion of a functor corresponds to the notion of such a partial algebra homomorphism. A covariant functor is a functor which preserves the directionality of an arrow in the domain category, whereas a contravariant functor is a functor which reverses it.

Each object $\mathcal{A}$ of a category $\mathfrak{A}$ determines a covariant functor $\mathbf{y}_{\mathcal{A}}: \mathfrak{A} \rightarrow$ Sets, called the covariant $H_{\mathfrak{A}}$-functor represented by $\mathcal{A}$, defined as follows:

i For all objects $\mathcal{X}$ in $\mathfrak{A}, \mathbf{y}_{\mathcal{A}}(\mathcal{X}):=\operatorname{Hom}_{\mathfrak{A}}(\mathcal{A}, \mathcal{X})$.

ii For all homomorphisms $f: \mathcal{X} \rightarrow \mathcal{Y}$ in $\mathfrak{A}$,

$$
\mathbf{y}_{\mathcal{A}}(f): \operatorname{Hom}_{\mathfrak{A}}(\mathcal{A}, \mathcal{X}) \rightarrow \operatorname{Hom}_{\mathfrak{A}}(\mathcal{A}, \mathcal{Y})
$$

is defined as post-composition with $f$, viz., $\mathbf{y}_{\mathcal{A}}(f)(g):=f \circ g$.

The covariant representable functor $\mathbf{y}_{\mathcal{A}}: \mathfrak{A} \rightarrow$ Sets, can be thought of as constructing an image of $\mathfrak{A}$ in Sets in a covariant way.

Now, let us consider the opposite category $\mathfrak{A}^{o p}$, and let $\mathcal{A}$ be an object in this category. Then, the contravariant $H_{\mathfrak{A}}$-functor represented by $\mathcal{A}$ is the contravariant functor $\mathbf{y}^{\mathcal{A}}: \mathfrak{A}^{o p} \rightarrow$ Sets , defined as follows:

i For all objects $\mathcal{B}$ in $\mathfrak{A}^{o p}, \mathbf{y}^{\mathcal{A}}(\mathcal{B}):=\operatorname{Hom}_{\mathfrak{A}^{o p}}(\mathcal{B}, \mathcal{A})$.

ii For all homomorphisms $f: \mathcal{C} \rightarrow \mathcal{B}$ in $\mathfrak{A}^{o p}$, 


$$
\mathbf{y}^{\mathcal{A}}(f): \operatorname{Hom}_{\mathfrak{Q}^{o p}}(\mathcal{B}, \mathcal{A}) \rightarrow \operatorname{Hom}_{\mathfrak{2 l}^{o p}}(\mathcal{C}, \mathcal{A})
$$

is defined as pre-composition with $f$, viz., $\mathbf{y}^{\mathcal{A}}(f)(g):=g \circ f$.

The contravariant $H_{\mathfrak{A}}$-functor represented by $\mathcal{A}$, viz. $\mathbf{y}^{\mathcal{A}}: \mathcal{F}^{o p} \rightarrow$ Sets, is called the functor of generalized elements (incoming homomorphisms) of $\mathcal{A}$. Moreover, the information contained in $\mathcal{A}$ is classified completely by its functor of generalized elements $\mathrm{y}^{\mathcal{A}}$. Dually the covariant $H_{\mathfrak{A}}$-functor represented by $\mathcal{A}$, viz. $\mathbf{y}_{\mathcal{A}}: \mathfrak{A} \rightarrow$ Sets is called the functor of generalized co-elements (outgoing homomorphisms) of $\mathcal{A}$. Similarly, the information contained in $\mathcal{A}$ is classified completely by its functor of generalized co-elements $\mathbf{y}_{\mathcal{A}}$.

Now, given a locally small category $\mathfrak{A}$, that is, a category such that for all objects $\mathcal{B}, \mathcal{A}$, the $\operatorname{Hom}$-class $\operatorname{Hom}_{\mathfrak{l}}(\mathcal{B}, \mathcal{A})$ is a set, we may consider the Hom $_{\mathfrak{A}}$-bifunctor:

$$
\operatorname{Hom}_{\mathfrak{A}}:=\mathbf{y}_{\mathcal{B}}^{\mathcal{A}}=\mathfrak{A}^{o p} \times \mathfrak{A} \rightarrow \text { Sets }
$$

from the product category $\mathfrak{A}^{o p} \times \mathfrak{A}$ to the category of sets, such that for objects $\mathcal{B}, \mathcal{A}$ of $\mathfrak{A}, \mathbf{y}_{\mathcal{B}}: \mathfrak{A} \rightarrow$ Sets is the covariant representable functor (represented by $\mathcal{B}$ ), and $\mathbf{y}^{\mathcal{A}}: \mathfrak{A}^{o p} \rightarrow$ Sets is the contravariant representable functor (represented by $\mathcal{A}$ ).

Continuing in the same frame of thought, the next question arising is the following: Which is the proper notion of morphism to capture the notion of a transformation from some functor to another functor having both the same domain and the same codomain categories? Defining the proper notion of morphism between such functors is important because it would allow us to legitimate the notion of a functor category $\mathfrak{C}^{\mathfrak{2}}$, where the algebraic objects would be functors $\mathbf{F}: \mathfrak{A} \rightarrow \mathfrak{C}$ and the morphisms would be the sought transformations between such functors.

The leading idea has to do with the requirement that a transformation of the sought form should compare two functorial processes having the same domain and the same codomain in a way that is not dependent on the specific objects and arrows involved, that is it should relate the processes themselves without the intervention of $a d$ hoc choices. This is precisely the notion required for the formalization of the concept of naturality referring to the relation or comparison of two 
functorial processes sharing the same source and the same target categories. Concomitantly the corresponding notion of morphism between functors of the above form is encapsulated in the notion of a natural transformation.

More precisely, if $\mathbf{F}, \mathbf{G}$, are functors from the category $\mathfrak{A}$ to the category $\mathfrak{C}$, a natural transformation $\tau$ from $\mathbf{F}$ to $\mathbf{G}$ is a function assigning to each object $\mathcal{A}$ in $\mathfrak{A}$ a morphism $\tau_{\mathcal{A}}$ from $\mathbf{F}(\mathcal{A})$ to $\mathbf{G}(\mathcal{A})$ in $\mathfrak{C}$, such that for every arrow $f: \mathcal{A} \rightarrow \mathcal{B}$ in $\mathfrak{A}$ the following diagram in $\mathfrak{C}$ commutes:

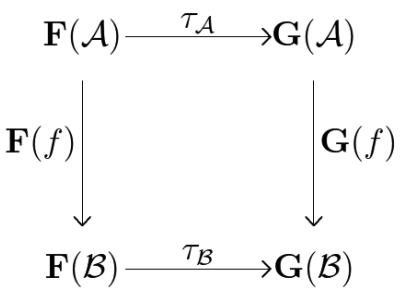

That is, for every arrow $f: \mathcal{A} \rightarrow \mathcal{B}$ in $\mathfrak{A}$ we have:

$$
\mathbf{G}(f) \circ \tau_{\mathcal{A}}=\tau_{\mathcal{B}} \circ \mathbf{F}(f)
$$

A natural transformation $\tau: \mathbf{F} \rightarrow \mathbf{G}$ is called a natural isomorphism (or natural equivalence) if every component $\tau_{\mathcal{A}}$ is invertible. Obviously, a natural isomorphism is an invertible natural transformation in the functor category $\mathfrak{C}^{\mathfrak{A}}$. A natural isomorphism of functors defines precisely the categorical means of metaphora. Paraphrasing Hermann Weyl, we may say that a science can only determine its domain of investigation up to a natural isomorphism. In particular, it remains quite indifferent as to the "essence" of its objects. In this manner, the notion of a natural isomorphism demarcates the insurmountable boundary of cognition, in the sense that through the disclosure of natural isomorphisms of functors it is possible to transfer any insights gained in one domain to the isomorphic domain.

This is a key concept and justifies a posteriori the whole categorical framework, since it captures precisely the criterion of naturality referring to the comparison of any two homoeoid functorial processes. For this purpose, it is instructive to adopt the following terminology:

Suppose that $\mathbf{F}(\mathcal{R})$ is an expression with an argument $\mathcal{R}$, such that given an object $\mathcal{A}$ in $\mathfrak{A}$, the expression $\mathbf{F}(\mathcal{A})$ holds for an 
object in $\mathfrak{C}$, and given an arrow $f$ in the set of incoming or outgoing arrows from $\mathcal{A}$ the expression $\mathbf{F}(f)$ holds for an arrow in $\mathfrak{C}$. We say that $\mathbf{F}(\mathcal{R})$ is functorial in $\mathcal{R}$, as $\mathcal{R}$ ranges over $\mathfrak{A}$, if this assignment yields a functor from $\mathfrak{A}$ to $\mathfrak{C}$. For example for a category $\mathfrak{A}$ the $\operatorname{Hom}_{\mathfrak{A}}$-set expression $\operatorname{Hom}_{\mathfrak{A}}(\mathcal{A}, \mathcal{B})$ is functorial in both $\mathcal{A}$ ranging over $\mathfrak{A}^{o p}$ and $\mathcal{B}$ ranging over $\mathfrak{A}$. Thus, the $\operatorname{Hom}_{\mathfrak{A}}$-set expression $\operatorname{Hom}_{\mathfrak{A}}(\mathcal{A}, \mathcal{B})$ is bifunctorial over the product category $\mathfrak{A}^{o p} \times \mathfrak{A}$. Moreover, we say that the expressions $\mathbf{F}_{1}(\mathcal{R}), \mathbf{F}_{2}(\mathcal{R})$ both functorial in $\mathcal{R}$, as $\mathcal{R}$ ranges over $\mathfrak{A}$, are naturally isomorphic in $\mathcal{R}$, or equivalently, that there exists an isomorphism natural in $\mathcal{R}$, if and only if there is a natural isomorphism between the functors $F_{1}$ and $\mathbf{F}_{2}$ from the category $\mathfrak{A}$ to the category $\mathfrak{C}$.

It is also useful to think of the category of all (locally small) categories and functors, denoted by Cat. In Cat for any two categories $\mathfrak{A}, \mathfrak{C}$, we define the operation of exponentiation as follows:

$$
\mathfrak{C}^{\mathfrak{A}}:=\operatorname{Functors}(\mathfrak{A}, \mathfrak{C})
$$

where Functors $(\mathfrak{A}, \mathfrak{C})$ is the category of functors from $\mathfrak{A}$ to $\mathfrak{C}$ and natural transformations between them. From the perspective of Cat the notion of natural transformation between functors allows to transform the $H_{\text {Cat }}$-set $\operatorname{Hom}_{\text {Cat }}(\mathfrak{A}, \mathfrak{C})$ into a category with exponentials. In this setting we define the evaluation functor:

$$
\mathbf{e v}: \mathfrak{C}^{\mathfrak{A}} \times \mathfrak{A} \rightarrow \mathfrak{C}
$$

such that for any category $\mathfrak{D}$ and bifunctor $\mathbf{G}: \mathfrak{D} \times \mathfrak{A} \rightarrow \mathfrak{C}$ there is a functor $\tilde{\mathbf{G}}: \mathfrak{D} \rightarrow \mathfrak{C}^{\mathfrak{A}}$ defined by transposition, such that:

$$
\mathbf{e v} \circ\left(\tilde{\mathbf{G}} \times \mathbf{1}_{\mathfrak{A}}\right)=\mathbf{G}
$$

This is clearly the case if we define the functor $\tilde{\mathbf{G}}$, given the bifunctor $\mathbf{G}: \mathfrak{D} \times \mathfrak{A} \rightarrow \mathfrak{C}$ as follows:

$$
\tilde{\mathbf{G}}(\mathcal{D})(\mathcal{A})=\mathbf{G}(\mathcal{D}, \mathcal{A})
$$


As a simple application we may take the product category $\mathfrak{D} \times \mathfrak{A}$ and consider the first projection:

$$
\mathfrak{D} \times \mathfrak{A} \rightarrow \mathfrak{D}
$$

Then, if we transpose it we get a functor:

$$
\Delta: \mathfrak{D} \rightarrow \mathfrak{D}^{\mathfrak{A}}
$$

For an object $\mathcal{D}$ in $\mathfrak{D}$, the functor $\Delta(\mathcal{D})$ is the constant $\mathcal{D}$-valued functor, which for any object $\mathcal{A}$ of $\mathfrak{A}$ gives the constant value $\mathcal{D}$, and for any arrow $f$ of $\mathfrak{A}$ gives the identity arrow on $\mathcal{D}$. AND DUALITY

Firstly, we introduce the notion of equivalence of two categories $\mathfrak{D}$ and $\mathfrak{A}$ as follows: An equivalence of two categories $\mathfrak{D}$ and $\mathfrak{A}$ is defined by means of a pair of oppositely directing functors;

$$
\begin{aligned}
& \mathbf{F}: \mathfrak{D} \rightarrow \mathfrak{A} \\
& \mathbf{G}: \mathfrak{A} \rightarrow \mathfrak{D}
\end{aligned}
$$

and a pair of natural isomorphisms;

$$
\begin{aligned}
\tau: \mathbf{1}_{\mathfrak{D}} & \rightarrow \mathbf{G} \circ \mathbf{F} \\
\circlearrowright: \mathbf{1}_{\mathfrak{A}} & \rightarrow \mathbf{F} \circ \mathbf{G}
\end{aligned}
$$

where $\tau$ is a natural isomorphism of the identity functor on $\mathfrak{D}$ in $\mathfrak{D}^{\mathfrak{D}}$ and $\circlearrowright$ is a natural isomorphism of the identity functor on $\mathfrak{A}$ in $\mathfrak{A}^{\mathfrak{A}}$. In this case, the functorial process $\mathbf{F}: \mathfrak{D} \rightarrow \mathfrak{A}$ is called inverse to the functorial process $\mathbf{G}: \mathfrak{A} \rightarrow \mathfrak{D}$.

The notion of equivalence of categories transcribes the concept of similarity, or analogia, in a categorical/functorial context. Furthermore, it makes precise the notion of duality between categories. More concretely, duality theorems can be expressed in the form $\mathfrak{A} \simeq \mathfrak{B}^{\text {op }}$, meaning that the category $\mathfrak{A}$ is equivalent to the opposite of the category $\mathfrak{B}$. The notion of duality between categories, formalized by means of an equivalence as above, is very important because it means that 
two categories related by a duality transformation are similar in the sense that they play the same functional role.

The interpretational consequences of this notion are far reaching and especially interesting from a physical viewpoint. We have argued previously that categories of algebraic objects can be considered as providing solutions to coordinatization processes (conceived within an associatively closed universe of discourse) arising from physical measurement problems. If a coordinatization process is modeled functorially as a functor $\mathbf{F}: \mathfrak{D} \rightarrow \mathfrak{A}$, which encodes the information of $\mathfrak{D}$ in terms of a category of algebraic objects $\mathfrak{A}$, then the existence of an inverse functorial process $\mathbf{G}: \mathfrak{A} \rightarrow \mathfrak{D}$ decodes the algebraic information of $\mathfrak{A}$ back into the terms of $\mathfrak{D}$, which in turn, is identified as the opposite or dual category of $\mathfrak{A}$.

In this way, a physical measurement problem presented in terms of a category of geometric objects $\mathfrak{D}$ can be resolved by finding a duality, that is by establishing a natural equivalence between a category of algebraic objects $\mathfrak{A}$ and the opposite of $\mathfrak{D}$. This frame of thought can be extrapolated in formal terms of duality between geometric objects and algebraic objects. Then, keeping in mind the encoding functionality of a functorial coordinatization process as well as the decoding functionality of its inverse (if it exists), we may interpret algebraic objects as syntactical objects and their dual geometric objects as semantical or phenomenological objects.

As a particular example we may cite the Stone duality established between the category of Boolean algebras (or equivalently Boolean unital rings) and the category of compact totally disconnected Hausdorff topological spaces (Stone spaces). In the finite case this duality restricts to an equivalence of the category of finite Boolean algebras and the opposite of the category of finite sets. In this way, each Boolean algebra $B$ has an associated topological space $S(B)$, called its Stone space. The points of the Stone space $S(B)$ are the ultrafilters of the Boolean algebra $B$, or equivalently the homomorphisms from $B$ to the 2 -element Boolean algebra. A basis of the topology of $S(B)$ consists of sets of the form $\{x \in S(B) \mid b \in x\}$ where $b \in B$. Conversely, given any topological space $X$, the collection of subsets of $X$ which are compact open or equivalently clopen, that is, both closed and open sets, is a Boolean algebra. Now, the assignment of a Boolean algebra to its Stone space is functorial, that is it corresponds to the object part of a contravariant functor from the category of Boolean algebras to the category of Stone spaces:

$$
\operatorname{Hom}_{\mathcal{B}}(-, 2): \mathcal{B}^{o p} \rightarrow \mathcal{T}
$$


where $\mathcal{B}, \mathcal{T}$ are the categories of Boolean algebras and Stone spaces correspondingly. The functor $\operatorname{Hom}_{\mathcal{B}}(-, 2)$ sends a Boolean algebra $B$ to the Stone space $S(B):=X_{B}$, and a Boolean algebra homomorphism $F: B \rightarrow A$ to the continuous function $\operatorname{Hom}_{\mathcal{B}}(F, 2)$, whose action is defined by precomposition with $F$, by which we mean that it sends $H: A \rightarrow 2$ of $X_{A}$ to $F \circ H: B \rightarrow 2$ of $X_{B}$. Now, there exists an inverse functor:

$$
\operatorname{Hom}_{\mathcal{T}}(-, 2): \mathcal{T} \rightarrow \mathcal{B}^{o p}
$$

which sends a Stone space $S$ to the set of continuous functions from $S$ to the Stone space 2 , which is endowed with the structure of a Boolean algebra, and further identified as the Boolean algebra of its clopen sets. Moreover, it sends a continuous function of Stone spaces $f: S_{1} \rightarrow S_{2}$ to the homomorphism of Boolean algebras $\operatorname{Hom}_{\mathcal{T}}(f, 2)$, whose action is defined by postcomposition with $f$ correspondingly.

Note that the duality between the category of Boolean algebras and the category of Stone spaces is based on a dual interpretation of the twoelement set 2 , or in other words; as the two-element trivial Boolean algebra, or again as a two-point Stone space. Essentially, the functorial process of coordinatization corresponding to a measurement problem of classical physics can be presented in the form of an information encoding functor $\operatorname{Hom}_{\mathcal{T}}(-, 2): \mathcal{T} \rightarrow \mathcal{B}^{o p}$, and is resolved by specifying the inverse information decoding functor $\operatorname{Hom}_{\mathcal{B}}(-, 2): \mathcal{B}^{o p} \rightarrow \mathcal{T}$ which establishes the equivalence of the geometric phenomenological category $\mathcal{T}$ with the dual of the algebraic category $\mathcal{B}$. Again from a physical viewpoint, the geometric category $\mathcal{T}$ is understood as a category of geometric spaces of physical states (geometric state spaces), whereas the algebraic category $\mathcal{B}$ providing an operational solution to the corresponding measurement problem is understood as a category of algebras of observables. Now, the distinguished two-element trivial Boolean algebra acts, from the perspective of the algebraic category, as an evaluation number-like object, that is, as a binary measurement device, for the evaluation of Boolean observables.

Concomitantly, the geometric state space observed by means of such a measurement procedure of observables is a Stone space, whose set of continuous functions into the Stone space 2 is identified as the Boolean algebra of its clopen sets. It is also important to notice that the above 
duality factors through the associatively closed universe of sets, employed as a scaffolding. This is also clear from the dual interpretation that the two-element set 2 acquires by its conceptualization within the dual categories of Boolean algebras and Stone spaces. From this fact we can draw two conclusions: Firstly, the category of sets plays the role of Aristotelian substance in the categorical framework. The substance acquires hypostatic form only in relation to a specific category of algebraic objects or its dual category of geometric objects (if it exists) and most importantly, this process of form-acquisition is functorial. Then, its form is conceived in a dual sense, meaning that its algebraic and geometric manifestations are understood as complementary aspects related by means of the algebraic/geometric object duality. Secondly, the category of sets, understood as a category of Aristotelian substance, and due to its functionality for the enunciation of algebraic/geometric dualities, should play a fundamental role in the representable elaboration of more complicated functorial coordinatization processes. These encoding processes may not have an exact inverse decoding process, but still the latter can be approximated functorially as will be explained below.

The purpose of explaining in detail the case of Stone duality, being the precursor of other dualities, such as the case of Gelfand duality, or Grothendieck duality, has to do with the fact that it is also related to logic. Of course, from the perspective of categorical logic, algebra is connected with logic, in the sense that logical theories may be understood from the perspective of algebraic categories.

In particular, Boolean algebras correspond to classical proposition theories which describe the measurement procedures of classical physical theories. Thus, the category of Boolean algebras can be considered as the category of logical propositional theories of classical physics, whereas its dual category of Stone spaces can be seen as the category of geometric state-spaces observed by means of two-valued measurement procedures of Boolean observables. Hence, the set of geometric state models corresponding to a Boolean algebra in a classical measurement situation is obtained by taking homomorphisms from this algebra to the twoelement Boolean algebra 2 , that is the functor:

$$
\operatorname{Hom}_{\mathcal{B}}(-, 2): \mathcal{B}^{o p} \rightarrow \text { Sets }
$$

evaluated at $B$ in $\mathcal{B}$, gives:

$$
\operatorname{Models}_{B} \simeq \operatorname{Hom}_{\mathcal{B}}(B, 2)
$$

Inversely, because of Stone duality, the definition of the appropriate topology on the set of geometric state models, gives a topological Stone 
space, by means of which we are able to retrieve the Boolean algebra $B$ by taking morphisms in the category of Stone spaces from that space into the Stone space 2 , that is:

$$
B \cong H o m_{\mathcal{T}}\left(\text { Models }_{B}, 2\right)
$$

Again, it is instructive to observe the dual role that the two-element set 2 plays in the algebraic (logical)/geometric (phenomenological) Stone duality. By analogia, it is a natural question to ask if such a dual role can be played not from a set, like 2 in the case of Stone duality, but from a whole category of sets or even a diagram in Sets.

For example, if we consider as a dualizing object the small category of sets Sets, then a logical theory should correspond to a category $\mathfrak{L}$, whereas the category of logical theories would be an appropriate category of categories. Then, the category of Sets-valued geometric state models of the logical theory $\mathfrak{L}$, would be a corresponding category of functors from $\mathfrak{L}$ to Sets, that is:

$$
\operatorname{Models}_{\mathfrak{L}} \simeq \operatorname{Functors}(\mathfrak{L}, \operatorname{Sets}) \simeq \operatorname{Sets}^{\mathfrak{L}}
$$

where Functors $(\mathfrak{L}$, Sets) is the category of functors from $\mathfrak{L}$ to Sets and natural transformations between them. Then, the logical theory $\mathfrak{L}$ could be retrieved from the category of geometric state models Models $_{\mathfrak{L}}$ as the category of suitable functors from Models $_{\mathfrak{L}}$ to Sets preserving the required properties, so that:

$$
\left.\mathfrak{L} \simeq \text { Functors(Models } \mathbf{L}_{\mathfrak{L}}, \text { Sets }\right)
$$

The exercise of treating a diagram in Sets as a dualizing object, and in particular, a diagram in the category of finite sets (being equivalent to the category of finite Boolean algebras) can be used as a natural starting point for the analysis of quantum logic from the perspective of functorial duality. In this way, the transition from classical measurement procedures to quantum measurement procedures can be understood operationally as a transition from a Boolean algebra to a categorical diagram of Boolean algebras. This physical viewpoint will be developed in detail as we progress. 
The concept of algebraic/geometric duality also pertains to the notion of physical coordinate systems, or more generally, physical reference frames. More precisely a global coordinate system $\Sigma$ can be understood as an isomorphism $\Sigma: M \rightarrow E$ between a geometric object $M$ in some class and a standard object $E$ in that class. A local coordinate system (such as the coordinate charts on a manifold) is an isomorphism between a local part of a geometric object in some class and a local part of a standard object in that class.

Thus, keeping in mind the distinction between local and global coordinate systems we may use as a standard object $E$ a real Euclidean $n$-dimensional topological vector space. So if we identify the standard (local) geometric object as $E=\mathbb{R}^{n}$, then a (local) coordinate system is a (local) isomorphism $\Sigma: M \rightarrow \mathbb{R}^{n}$. We can think of this (local) isomorphism as related with an algebraic/geometric duality, where the algebraic object is identified as an algebra of observables (corresponding to a (local) measurement procedure) evaluated on a dualizing object, such that the (local) observable geometric state models of this algebra constitute the standard geometric object $E$.

In the particular case that $E=\mathbb{R}^{n}$, we can see immediately that $\mathbb{R}^{n} \cong \operatorname{Hom}_{\mathcal{C}^{\infty}}\left(\mathcal{C}^{\infty}{ }_{\mathbb{R}^{n}}, \mathbb{R}\right)$. Thus, the set $\mathbb{R}$ plays the role of a dualizing object, interpreted algebraically as an $\mathbb{R}$-algebra (field), and geometrically as a Euclidean space. Hence, the set of geometric state models corresponding to the algebra of smooth functions $\mathcal{C}_{\mathbb{R}^{n}}^{\infty}$ in a classical smooth measurement situation is obtained by taking homomorphisms from this algebra to the field $\mathbb{R}$, that is:

$$
\text { Models }_{\mathcal{C}_{\mathbb{R}^{n}}} \simeq \operatorname{Hom}_{\mathcal{C}^{\infty}}\left(\mathcal{C}_{\mathbb{R}^{n}}^{\infty}, R\right)
$$

Inversely, the set of geometric state models viewed as the Euclidean space $E=\mathbb{R}^{n}$, allows us to retrieve $\mathcal{C}_{\mathbb{R}^{n}}^{\infty}$ by taking morphisms in the category of Euclidean spaces from that space into the Euclidean space $\mathbb{R}$, that is:

$$
\mathcal{C}_{\mathbb{R}^{n}}^{\infty} \cong \operatorname{Hom}_{\mathcal{E}}\left(\text { Models }_{\mathcal{C}^{\infty}}, \mathbb{R}\right)
$$

We have concluded above that the definition of a (local) coordinate system (for the example mentioned) is understood as a (local) isomorphism: 


\section{$\Sigma: M \rightarrow$ Models $_{C^{\infty}}$}

Thus, generally a (local) coordinate system identifies (in the sense of similarity induced by an isomorphism) a geometric object (locally) with a standard "number-like" object (like $\mathbb{R}^{n}$ ), which is understood as a (local) space of geometric state models observed by means of evaluating an algebra of observables on a dualizing object (like the real numbers $\mathbb{R}$ ) in the sense of functorial duality. Note that generally, the identification induced by a (local) coordinate system is not natural. This means that we may legitimately define a whole class of (local) coordinate systems depending on the operational means of observation. In this class, we may define an equivalence relation partitioning it into equivalence classes of (local) coordinate systems.

Thus, arises the necessity to adopt a coordinate-invariant point of view, meaning that every expression formed in the descriptive terms of a (local) coordinate system should remain invariant under a transformation of this coordinate system into another in the same equivalence class. Equally, given a (local) coordinate system $\Sigma: M \rightarrow$ Models $_{A}$, where $A$ is an algebra of observables suited to a measurement procedure, and identifying by duality $Y \cong$ Models $_{A}$, then by an isomorphism $I: Y \rightarrow Y$ of the standard "number-like" object $Y$, we obtain a new (local) coordinate system of the geometric object $M$ in the equivalence class of $Y$, that is, $I \circ \Sigma: M \rightarrow Y$ by composition of the two (local) isomorphisms. Inversely, every other (local) coordinate system of $M, \Theta: M \rightarrow Y$, is obtained in this way. Accordingly, we obtain a group of isomorphisms of $Y$, denoted as $G:=I s o(Y)$, called the gauge group of the class of geometric objects.

Now, instead of taking a single geometric object, we may consider an indexed family of geometric objects. The indexing object, in the simplest case, is a set of parameters making a parameter space, or base space, over which the variation or parametrization of the family of geometric objects is conceived. This is equivalent to considering a discrete diagram of geometric objects in some appropriate category. Note that, the indexing object might be a category itself in the most general case. For example, if the indexing category is a set $X$ which parameterizes geometric objects in the category of sets, then the functor category Sets $^{X}$ is the category of $X$-indexed sets (category of discrete diagrams of sets). So the objects of Sets $^{X}$ are $X$-indexed families of sets $\left(A_{x}\right)_{x \in X}$ and the morphisms are $X$-indexed families of functions: 


$$
\left(f_{i}: A_{x} \rightarrow B_{x}\right)_{x \in X}:\left(A_{x}\right)_{x \in X} \rightarrow\left(B_{x}\right)_{x \in X}
$$

The functor category Sets $^{X}$ can be described equivalently as the "comma", or slice category of sets Sets over the indexing set $X$, denoted by Sets $/ X$. In this category, the objects are the morphisms $\alpha: A \rightarrow X$ and the arrows are the commutative triangles over the indexing set $X$. This is a very important example of an equivalence of categories, that is:

$$
\text { Sets }^{X} \simeq \text { Sets } / X
$$

The above functorial equivalence is described by means of the following inverse functors:

$$
\begin{aligned}
& \text { F : } \text { Sets }^{X} \rightarrow \text { Sets } / X \\
& \text { G : Sets } / X \rightarrow \text { Sets }^{X}
\end{aligned}
$$

such that:

$$
\mathbf{F}\left(\left(A_{x}\right)_{x \in X}\right)=\pi: \sum_{x} A_{x} \rightarrow X
$$

called the indexing projection morphism, and in the opposite direction:

$$
\mathbf{G}(\alpha: A \rightarrow X)=\left(\alpha^{-1}\{x\}\right)_{x \in X}
$$

The advantage of the functorially equivalent slice category Sets $/ X$ where $X$ is an indexing set (base space), is that it conforms with the intuition of a $X$-indexed family of objects of the category Sets, or in short, an $X$-indexed family of sets (considered as geometric objects). Moreover, the slice category $\mathfrak{X} / X$ is meaningful for any object $X$ in an arbitrary category (of geometric objects) $\mathfrak{X}$ conforming also with the intuition of an $X$-indexed family of objects of the category $\mathfrak{X}$. Now, if the category $\mathfrak{X}$ is complete, and in particular, it has pullbacks, then the process of re-parameterization along an arrow $h: X \rightarrow Y$ in $\mathfrak{X}$ is represented by the pullback (limit) functor:

$$
h^{*}: \mathfrak{X} / Y \rightarrow \mathfrak{X} / X
$$


In more familiar physical terms an $X$-indexed family of sets $\left(A_{x}\right)_{x \in X}$ (discrete diagram of sets) constitutes a fiber bundle, where the fibers $A_{x}$ are sets and $X$ is the base space of the bundle. Usually, the fibers have some extra structure, and topological or geometrical or differential compatibility conditions pertain between the fibers depending on the corresponding specification of the base space $X$. This is due to the natural requirement that the kind of variations in the fiber should conform to the kind of variations on the corresponding base point.

The crucial observation is that each fiber $A_{x}$ in a fiber bundle of set-theoretic geometric objects, that is to say each object $A_{x}$ in an $X_{\text {- }}$ indexed family of set-theoretic geometric objects $\left(A_{x}\right)_{x \in X}$, by virtue of belonging to a certain class of geometric objects, can be legitimately identified with a standard "number-like" object $E$ in that class. This is possible by means of a parameterizing point $x$-based coordinate system, expressed in terms of a point $x$-based isomorphism $\Sigma_{x}: A_{x} \rightarrow E$.

In this way, we obtain a separate coordinate system for each base point $x$ in the base space $X$. The complete family $\left(\Sigma_{x}\right)_{x \in X}$ (conforming with the corresponding topological, geometrical or differential compatibility conditions), if it exists, constitutes a global gauge, or global trivialization, for the fiber bundle $\left(A_{x}\right)_{x \in X}$. Correspondingly, a local gauge refers to a local trivialization for the fiber bundle $\left(A_{x}\right)_{x \in X}$ obtained in the same manner. Usually, a global gauge is not possible or available, but local gauges do exist, trivializing only parts of a fiber bundle in a consistent way. Note that the trivial bundle has the same fiber $E$ for each point $x \in X$, denoted as $(E)_{x \in X}$. Then, a function $l: X \rightarrow E$ has a graph $\{(x, l(x)): x \in X\} \subset X \times E$. The analogous notion to the graph of a function $l: X \rightarrow E$ referring to the trivial bundle $(E)_{x \in X}$ is the notion of section referring to an arbitrary fiber bundle.

In this sense, a (local) section of a fiber bundle may be thought of as a collection of representative elements $s=\left(s_{x}\right)_{x \in X}$ from the fibers of the fiber bundle $\left(A_{x}\right)_{x \in X}$. It is precisely sections of a fiber bundle that can be represented locally or globally in terms of "number-like" quantities by means of a local or global gauge correspondingly. Thus, a (local) gauge is a (local) fiber bundle isomorphism $\Sigma$ from the bundle $\left(A_{x}\right)_{x \in X}$ to the 
trivial bundle $(E)_{x \in X}$. Now, analogously to the case of (local) coordinate systems of single geometric objects, every family of (local) isomorphisms $\left(I_{x}\right)_{x \in X}$ of $(E)_{x \in X}$, provides a new (local) gauge $\left(I_{x} \circ \Sigma_{x}\right)_{x \in X}$, thus transforming the (local) gauge $\left(\Sigma_{x}\right)_{x \in X}$ into the (local) gauge $\left(I_{x} \circ \Sigma_{x}\right)_{x \in X}$. In this case we say that $\left(I_{x}\right)_{x \in X}$ constitutes a (local) gauge transformation.

We may conclude the above discussion by noticing that the notion of a gauge corresponds to the notion of a varying coordinate system. In the example of fiber bundles presented above, the variation is over a parameter space (base space), such that the variation of coordinate system is dependent on the location of an observer occupying a point $x$ of the base space. In the context of the same example, a gauge transformation is a change of coordinate system applied to each such location $x$ of the base space $X$.

From a categorical perspective, this is an example of a gauge theory formulated within the category of discrete diagrams of sets, namely the functor category Sets $^{X}$, or equivalently, the category of $X$-indexed sets Sets / $X$ (or other set-theoretic geometric objects). The idea of gauge should not depend on the indexing object used for its manifestation, meaning that the indexing object might be a category itself. As an example, we may think of a partially ordered set, considered as a category with arrows being inclusions, corresponding to the partial order of the open sets on a topological space. Then, it becomes possible to formulate a gauge theory within the category of diagrams of sets indexed (or localized) by the category of open sets on a topological space. We will see later that this functor category is technically called the category of (pre)sheaves (of sets) on a topological space $X$, denoted by $\operatorname{Sets}^{\mathcal{O}(X)^{o p}}$.

The analogy with the previous case is going to become evident after we prove that we may formulate the theory equivalently within the category of (étale) topological bundles, which is the category of $\mathcal{O}(X)$ indexed topological spaces denoted by $\operatorname{Top} / X$. Therefore, when topology is involved it is appropriate to formulate the notion of a gauge theory within the category $\operatorname{Sets}^{\mathcal{O}(X)^{o p}}$, where $\mathcal{O}(X)$ is the indexing (localizing) category of open sets in the topology of $X$.

Hence, in the general case, where the indexing or localization is defined by a category, the notion of a gauge is that of a coordinate system (or more generally a reference frame) that varies depending on the base object of the indexing category over which it is defined. Then, a gauge transformation is a change of reference frame applied to each such base object of the indexing category, and a gauge theory is a theory describing 
the behavior of a physical system to which such gauge transformations can be applied. Concomitantly, the physical requirement of gauge invariance is a statement of the fact that all physical observable attributes should transform naturally (or remain invariant) under gauge transformations.

In order to understand the notion of a gauge within a general category of the form $\operatorname{Sets}^{\mathfrak{A}^{o p}}$, where $\mathfrak{A}$ is an indexing or localizing category, it is instructive to recall that the notion of a (local) gauge (coordinate system) for a set-theoretic geometric object in a class is understood as a (local) isomorphism with a standard geometric object in the same class:

$$
\Sigma: G \rightarrow \text { Models }_{\mathcal{A}}
$$

where the set of geometric state models (geometric state-space representing the standard geometric object) corresponding to a coordinatizing algebra of observables in a measurement procedure, is obtained by taking homomorphisms from this algebra to some appropriate dualizing object in the same category $\mathfrak{A}$, denoted by $\mathcal{D}$ (like the real numbers $\mathbb{R}$ ), that is:

$$
\text { Models }_{\mathcal{A}} \simeq \operatorname{Hom}_{\mathfrak{A}}(\mathcal{A}, \mathcal{D})
$$

Thus, generally a (local) coordinate system identifies (in the sense of similarity induced by an isomorphism) a geometric object (locally) with a standard geometric object, which is understood as a (local) space of geometric state models observed by means of evaluating an algebra of observables on a dualizing object in the sense of functorial duality. Now, a natural question arising by analogia is the following: If the notion of a geometric object $G$ in a class, is substituted by the notion of a contravariant functor $\mathbf{G}: \mathfrak{A}^{o p} \rightarrow$ Sets in a functor category Sets $\mathfrak{A d}^{\mathfrak{A}^{o p}}$, or by the notion of a covariant functor $\mathbf{G}: \mathfrak{A} \rightarrow$ Sets in a functor category $\operatorname{Sets}^{\mathfrak{A}}$, where $\mathfrak{A}$ is an appropriate category of algebras of observables, then can we define a corresponding notion of a functorial gauge for the functor $\mathbf{G}$ ?

Intuitively, the notion of a functorial (local) gauge for $\mathbf{G}$ would be a (local) natural isomorphism with a standard object in the same category. Again, this standard functor would correspond to a functorial geometric state space, obtained as a category of functorial state models corresponding to a coordinatizing algebras of observables $\mathcal{A}$, that is: 


$$
\text { FModels }_{\mathcal{A}} \simeq \operatorname{Hom}_{\mathfrak{A}}(\mathcal{A},-)
$$

or equivalently, in the dual formulation:

$$
\text { FModels }_{\mathcal{A}} \simeq \operatorname{Hom}_{\mathfrak{2 l}^{o p}}(-, \mathcal{A})
$$

where (-) is a varying algebraic object in $\mathfrak{A}$ (to be thought of as a varying dualizing object). Thus, intuitively a functorial (local) gauge of $\mathbf{G}: \mathfrak{A}^{o p} \rightarrow$ Sets should be expressed as a (local) natural isomorphism (invertible natural transformation) of Sets -valued functors:

$$
\mathbf{G} \cong \operatorname{Hom}_{\mathfrak{A}^{o p}}(-, \mathcal{A})
$$

when $\mathbf{G}$ is a contravariant Sets -valued functor, and dually:

$$
\mathbf{G} \cong \operatorname{Hom}_{\mathfrak{A}}(\mathcal{A},-)
$$

when $\mathbf{G}$ is a covariant Sets -valued functor. One might ask, why we have restricted our attention to the case of Sets -valued functors. The reason is that the case of Sets -valued functors is enough in order to perform the corresponding gauge formulation in the general case of functors valued in an arbitrary category, using the notion of parameterized functorial gauge. Conceptually, we have already seen the role that the category of Sets plays in the case of functorial duality to enable factorization of duality through the scaffolding of the category of Sets, interpreted as a category of Aristotelian substance.

Before expanding on the notion of a functorial gauge for a functor of the form $\mathbf{G}: \mathfrak{A}^{o p} \rightarrow$ Sets it is essential to provide a brief overview of the functor category $\operatorname{Sets}^{\mathfrak{2}^{o p}}$, where $\mathfrak{A}$ is an indexing or localizing category. The functor category $\operatorname{Sets}^{2 \mathfrak{2}^{o p}}$ may be thought of as the category of diagrams on the indexing category $\mathfrak{A}$. Technically, it is called the category of presheaves on $\mathfrak{A}$.

For a category $\mathfrak{A}$ the functor category of presheaves (of sets) $\operatorname{Sets}^{2^{20 p}}$ is the category of all contravariant functors from $\mathfrak{A}$ to Sets and all natural transformations between them. $\mathrm{A}$ functor $\mathrm{P}$ is a 
structure-preserving morphism of these categories, that is, it preserves composition and identities. A presheaf functor in the category $\operatorname{Sets}^{\mathfrak{A l}^{\text {op }}}$ can be thought of as constructing an image of $\mathfrak{A}$ in Sets contravariantly, or as a contravariant translation of the language of $\mathfrak{A}$ into that of Sets.

Given another such translation (contravariant functor) $\mathbf{Q}$ of $\mathfrak{A}$ into Sets we need to compare them. This can be done by giving, for each object $\mathcal{A}$ in $\mathfrak{A}$ a transformation $T_{\mathcal{A}}: \mathbf{P}(\mathcal{A}) \rightarrow \mathbf{Q}(\mathcal{A})$ which compares the two images of the object $\mathcal{A}$. Not any morphism will do, however, as we would like the construction to be parametric in $\mathcal{A}$, rather than ad hoc. Since $\mathcal{A}$ is an object in $\mathfrak{A}$ while $\mathbf{P}(\mathcal{A})$ is in Sets we cannot link them by a morphism. Rather, the goal is that the transformation should respect the morphisms of $\mathfrak{A}$, or in other words, the interpretations of $v: \mathcal{C} \rightarrow \mathcal{A}$ by $\mathbf{P}$ and $\mathbf{Q}$ should be compatible with the transformation under $T$. Thus, $T$ is a natural transformation in the category of presheaves $\operatorname{Sets}^{\mathfrak{A l}^{o p}}$.

An object $\mathbf{P}$ of Sets $^{\mathfrak{2 l}^{\text {op }}}$ may be understood as a left categorical action of $\mathfrak{A}^{o p}$ on a set which is partitioned into sorts parameterized by the objects of $\mathfrak{A}$, and such that whenever $v: \mathcal{C} \rightarrow \mathcal{A}$ is an arrow and $p$ is an element of $\mathbf{P}$ of sort $\mathcal{A}$, then the pullback of $p$ along $v: \mathcal{C} \rightarrow \mathcal{A}$, denoted by $p \cdot v$, is specified as an element of $\mathrm{P}$ of sort $\mathcal{C}$. Such a left action $\mathbf{P}$ is referred as a $\mathfrak{A}^{o p}$-variable set.

The category of elements of a presheaf functor $\mathbf{P}$ (or categorical diagram of a presheaf functor $\mathbf{P})$, denoted by $\int(\mathbf{P}, \mathfrak{A})$, is defined as follows: Its objects are all pairs $(\mathcal{A}, p)$, and its morphisms $\left(\mathcal{A}^{\prime}, p^{\prime}\right) \rightarrow(\mathcal{A}, p)$ are those morphisms $u: \mathcal{A}^{\prime} \rightarrow \mathcal{A}$ of $\mathfrak{A}$ for which $p \cdot u=p^{\prime}$. Projection on the second coordinate of $\int(\mathbf{P}, \mathfrak{A})$, defines a functor:

$$
\int_{\mathbf{P}}: \int(\mathbf{P}, \mathfrak{A}) \rightarrow \mathfrak{A}
$$

$\int(\mathbf{P}, \mathfrak{A})$ together with the projection functor $\int_{\mathbf{P}}$ is called the split discrete fibration induced by $\mathbf{P}$, and $\mathfrak{A}$ is the base indexing or localizing category of the fibration. We note that the fibers are categories in which the only arrows are identity arrows. If $\mathcal{A}$ is an object of $\mathfrak{A}$, 
the inverse image under $\int_{\mathbf{P}}$ of $\mathcal{A}$ is simply the set $\mathbf{P}(\mathcal{A})$, although its elements are written as pairs so as to form a disjoint union.

Similarly, for a category $\mathfrak{A}$ the functor category of copresheaves (of sets) $\operatorname{Sets}^{\mathfrak{A}}$ is defined as the category of all covariant functors from $\mathfrak{A}$ to Sets and all natural transformations between them. Analogously, a copresheaf $\mathrm{P}$ may be understood as a right action of $\mathfrak{A}$ on a set which is partitioned into sorts parametrized by the objects of $\mathfrak{A}$ and such that whenever $v: \mathcal{C} \rightarrow \mathcal{A}$ is an arrow and $p$ is an element of $\mathrm{P}$ of sort $\mathcal{C}$, then $v \cdot p$ is specified as an element of $\mathrm{P}$ of sort $\mathcal{A}$. Such a right action $\mathrm{P}$ is referred as a $\mathfrak{A}$-variable set. The category of elements of a copresheaf functor $P$ (or diagram of a copresheaf functor $\mathbf{P})$, denoted by $\int(\mathbf{P}, \mathfrak{A})$ is defined as follows: Its objects are all pairs $(\mathcal{A}, p)$, and its morphisms $\left(\mathcal{A}^{\prime}, p^{\prime}\right) \rightarrow(\mathcal{A}, p)$ are those morphisms $u: \mathcal{A}^{\prime} \rightarrow \mathcal{A}$ of $\mathfrak{A}$ for which $u \cdot p^{\prime}=p$.

Notice that in both the functor categories of presheaves and copresheaves of sets standard objects are present. These are correspondingly the following:

i The contravariant $H_{\mathfrak{A}}$-functor represented by $\mathcal{A}$, that is:

$$
\mathbf{y}^{\mathcal{A}}=\operatorname{Hom}_{\mathfrak{A}^{o p}}(-, \mathcal{A}): \mathfrak{A}^{o p} \rightarrow \text { Sets }
$$

called the functor of generalized elements (incoming morphisms) of $\mathcal{A}$, which classifies completely the information contained in $\mathcal{A}$.

ii The covariant $H_{0}$-functor represented by $\mathcal{A}$, that is:

$$
\mathbf{y}_{\mathcal{A}}=\operatorname{Hom}_{\mathfrak{A}}(\mathcal{A},-): \mathfrak{A} \rightarrow \text { Sets }
$$

called the functor of generalized co-elements (outgoing morphisms) of $\mathcal{A}$. Similarly, the information contained in $\mathcal{A}$ is classified completely by its functor of generalized co-elements $\mathbf{y}_{\mathcal{A}}$.

In this way, we obtain simultaneously both a contravariant functor $\mathbf{y}^{(-)}$ and a covariant functor $\mathbf{y}_{(-)}$valued on the category of copresheaves and presheaves (of sets) correspondingly as follows: 


$$
\begin{aligned}
& \mathbf{y}^{(-)}: \mathfrak{A}^{o p} \rightarrow \operatorname{Sets}^{\mathfrak{A}} \\
& \mathbf{y}_{(-)}: \mathfrak{A} \rightarrow \operatorname{Sets}^{\mathfrak{A}^{o p}}
\end{aligned}
$$

which constitute the exponential transposes of the Hom $_{\mathfrak{A}}$-bifunctor:

$$
\operatorname{Hom}_{\mathfrak{A}}: \mathfrak{A}^{o p} \times \mathfrak{A} \rightarrow \text { Sets }
$$

from the product category $\mathfrak{A}^{o p} \times \mathfrak{A}$ to the category of Sets with respect to each of its arguments.

The covariant functor $\mathbf{y}_{(-)}: \mathfrak{A} \rightarrow \operatorname{Sets}^{\mathfrak{2}^{o p}}$ realizes the Nobuo Yoneda embedding of the category $\mathfrak{A}$ into the category of presheaves Sets $\mathfrak{2}^{\text {op }}$. The term embedding means that the functor $\mathbf{y}_{(-)}$is bijective when restricted to each set of morphisms with a given domain and codomain, and that it is injective on objects as well. The Yoneda embedding constitutes a representation of the category $\mathfrak{A}$ within the functor category of presheaves (of sets) $\operatorname{Sets}^{\mathfrak{A l}^{\text {op }}}$. In detail we have:

$$
\begin{gathered}
\mathbf{y}_{(-)}(\mathcal{A})=\mathbf{y}^{\mathcal{A}}=\operatorname{Hom}_{\mathfrak{A}^{o p}}(-, \mathcal{A}): \mathfrak{A}^{o p} \rightarrow \text { Sets } \\
\mathbf{y}_{(-)}(f)=\mathbf{y}^{f}: \operatorname{Hom}_{\mathfrak{A l}^{o p}}(-, \mathcal{A}) \rightarrow \operatorname{Hom}_{\mathfrak{A l}^{o p}}(-, \mathcal{C})
\end{gathered}
$$

where $f: \mathcal{A} \rightarrow \mathcal{C}$ is a morphism in $\mathfrak{A}$.

The importance of the Yoneda embedding is manifested by the so called Yoneda lemma, according to which, for any object $\mathcal{A}$ in a (locally) small category $\mathfrak{A}$ and any presheaf functor $\mathbf{P}$ in $\operatorname{Sets}^{\mathfrak{A}^{\text {op }}}$ there exists an isomorphism:

$$
\operatorname{Hom}_{\text {Sets }}^{\mathfrak{A L}^{o p}}\left(\mathbf{y}^{\mathcal{A}}, \mathbf{P}\right) \cong \mathbf{P}(\mathcal{A})
$$

written equivalently as:

$$
\operatorname{Nat}\left(\mathbf{y}^{\mathcal{A}}, \mathbf{P}\right) \cong \mathbf{P}(\mathcal{A})
$$

which is natural in both $\mathrm{P}$ and $\mathcal{A}$.

The main application of the Yoneda lemma is the following: Given objects $\mathcal{A}$ and $\mathcal{B}$ in a (locally) small category $\mathfrak{A}$, if $\mathbf{y}_{(-)}(\mathcal{A}) \cong \mathbf{y}_{(-)}(\mathcal{B})$, or equivalently, $\mathbf{y}^{\mathcal{A}} \cong \mathbf{y}^{\mathcal{B}}$, then $\mathcal{A} \cong \mathcal{B}$. 
The advantage of working within the category of presheaves Sets $\mathfrak{A d P}^{o p}$ instead of the category $\mathfrak{A}$ is that it is both complete (has all small limits) and cocomplete (has all small colimits), and most importantly, this is so because the category of Sets is both complete and cocomplete. Moreover, for every object $\mathcal{A}$ of $\mathfrak{A}$ the evaluation functor at $\mathcal{A}$, that is:

$$
\mathbf{e v}_{\mathcal{A}}: \operatorname{Sets}^{\mathfrak{A}^{o p}} \rightarrow \text { Sets }
$$

preserves both all limits and colimits.

Let us now return to the problem of understanding the notion of a functorial gauge (functorial reference frame) for a functor $\mathbf{G}: \mathfrak{A}^{o p} \rightarrow$ Sets, where $\mathfrak{A}$ is considered to be an appropriate category of coordinatizing algebras of observables. We have argued previously that a functorial gauge of $\mathbf{G}: \mathfrak{A}^{o p} \rightarrow$ Sets should be expressed as a natural isomorphism (invertible natural transformation) of Sets -valued functors:

$$
\mathbf{G} \cong \mathbf{H o m}_{\mathfrak{A}^{o p}}(-, \mathcal{A})
$$

where, the standard functor $\operatorname{Hom}_{\mathfrak{A}^{o p}}(-, \mathcal{A})$ in the category of presheaves $\operatorname{Sets}^{\mathfrak{A L}^{o p}}$ is physically equivalent to the functorial geometric state models of an algebra of observables $\mathcal{A}$, that is:

$$
\text { FModels }_{\mathcal{A}} \simeq \operatorname{Hom}_{\mathfrak{A l}^{o p}}(-, \mathcal{A})
$$

In categorical terminology, we find the notion of representability of a (contravariant or covariant) Sets-valued functor, defined as follows: A representation of a contravariant Sets -valued functor of the form $\mathbf{G}: \mathfrak{A}^{o p} \rightarrow$ Sets, where $\mathfrak{A}$ is a (locally) small category, consists of an object $\mathcal{A}$ in $\mathfrak{A}$ and a natural isomorphism:

$$
\operatorname{Hom}_{\mathfrak{A l}^{o p}}(-, \mathcal{A}) \cong \mathbf{G}
$$

or equivalently:

$$
\mathbf{y}^{\mathcal{A}} \cong \mathbf{G}
$$


where $\mathcal{A}$ is called the representing object of the functor $\mathbf{G}: \mathfrak{A}^{o p} \rightarrow$ Sets . Thus, $\mathbf{G}$ is representable, if and only if such a representing object exists. Note that, representations of contravariant Sets -valued functors are unique up to a unique isomorphism. Evidently, a dual formulation obtains, referring to a representation of a covariant Sets -valued functor of the form $\mathbf{G}: \mathfrak{A} \rightarrow$ Sets .

To be specific, the covariant functor $\mathbf{G}$ is representable if and only if there exists an object $\mathcal{A}$ in $\mathfrak{A}$ and a natural isomorphism:

$$
\operatorname{Hom}_{\mathfrak{A}}(\mathcal{A},-) \cong \mathbf{G}
$$

or equivalently:

$$
\mathbf{y}_{\mathcal{A}} \cong \mathbf{G}
$$

It follows immediately from the above that the categorical notion of representability of a Sets -valued functor is selfsame as the physical notion of a functorial gauge (functorial reference frame) of a Sets valued functor in the intended interpretation.

Furthermore, if we use the Yoneda lemma, we have:

$$
\operatorname{Nat}\left(\mathbf{y}^{\mathcal{A}}, \mathbf{G}\right) \cong \mathbf{G}(\mathcal{A})
$$

in the contravariant case, which is natural in both $\mathbf{G}$ and $\mathcal{A}$, and dually:

$$
\operatorname{Nat}\left(\mathbf{y}_{\mathcal{A}}, \mathbf{G}\right) \cong \mathbf{G}(\mathcal{A})
$$

in the covariant case, which is natural in both $\mathbf{G}$ and $\mathcal{A}$.

If we consider, for instance, the covariant case in more detail, then if $\Upsilon$ is a natural transformation in $\operatorname{Nat}\left(\mathbf{y}_{\mathcal{A}}, \mathbf{G}\right)$, that is:

$$
\Upsilon: \mathbf{y}_{\mathcal{A}} \rightarrow \mathbf{G}
$$

the element $y$ in $\mathbf{G}(\mathcal{A})$ corresponding to $\Upsilon$ is defined by:

$$
y=\Upsilon_{\mathcal{A}}\left(i d_{\mathcal{A}}\right)
$$


In the inverse direction, given any element $y$ in $\mathbf{G}(\mathcal{A})$ we define a natural transformation $\Upsilon^{y}: \mathbf{y}_{\mathcal{A}} \rightarrow \mathbf{G}$ as follows:

$$
\Upsilon_{\mathcal{D}}^{y}(h)=(\mathbf{G}(h))(y)=h \cdot y
$$

where $h$ is a generalized co-element of $\mathcal{A}$, which means $h$ belongs to $\operatorname{Hom}_{\mathfrak{A}}(\mathcal{A}, D)$. Thus, as a consequence of the Yoneda lemma, given any element $y$ in $\mathbf{G}(\mathcal{A})$ we need to know when the induced natural transformation $\Upsilon^{y}: \mathbf{y}_{\mathcal{A}} \rightarrow \mathbf{G}$ is a natural isomorphism, because in this case the covariant Sets -valued functor $\mathbf{G}$ is representable, or equivalently, there exists a functorial gauge for $\mathbf{G}$.

This is actually the case if and only if there exists a pair $(\mathcal{A}, y)$ in $\int(\mathbf{P}, \mathfrak{A})$, where $\mathcal{A}$ in $\mathfrak{A}$ and $y$ in $\mathbf{G}(\mathcal{A})$, such that, for every pair $(\mathcal{D}, z)$ with $z$ in $\mathbf{G}(\mathcal{D})$, a unique morphism $h: \mathcal{A} \rightarrow \mathcal{D}$ pertains, such that $(\mathbf{G}(h))(y)=h \cdot y=z$. This is technically called a universal element of $\mathbf{G}$ and is interpreted as a universal gauge of $\mathbf{G}$. A universal gauge $(\mathcal{A}, y)$ of $\mathbf{G}$ actually acts as an initial object in $\int(\mathbf{G}, \mathfrak{A})$. Thus, we conclude that given any element $y$ in $\mathbf{G}(\mathcal{A})$ the induced natural transformation $\Upsilon^{y}: \mathbf{y}_{\mathcal{A}} \rightarrow \mathbf{G}$ is a natural isomorphism, if and only if $(\mathcal{A}, y)$ is a universal gauge of $\mathbf{G}$. Hence, representations, or functorial gauges (functorial reference frames) of $\mathbf{G}$, that is, natural isomorphisms $\mathbf{y}_{\mathcal{A}} \cong \mathbf{G}$, are in bijective correspondence with universal gauges $(\mathcal{A}, y)$ of G.

Precisely analogous conclusions hold in the dual case of a contravariant Sets -valued functor $\mathbf{G}$, that is, representations or functorial gauges (functorial reference frames) of $\mathbf{G}$, or natural isomorphisms $\mathbf{y}^{\mathcal{A}} \cong \mathbf{G}$, are in bijective correspondence with universal gauges $(\mathcal{A}, y)$ of $\mathbf{G}$, where a universal gauge $(\mathcal{A}, y)$ of $\mathbf{G}$ acts now as a terminal object in $\int(\mathbf{G}, \mathfrak{A})$. Note that in the intended physical interpretation, a universal gauge of $\mathbf{G}$ is provided by a pair $(\mathcal{A}, y)$, where $\mathcal{A}$ is a coordinatizing algebra in $\mathfrak{A}$, and $y$ in $\mathbf{G}(\mathcal{A})$, which is acting as an initial (in the covariant case) or as a terminal (in the contravariant case) object in the corresponding category of elements $\int(\mathbf{G}, \mathfrak{A})$. 
We have seen previously that the problem of finding a functorial gauge for (a covariant or contravariant) Sets-valued functor $\mathbf{G}$ is resolved by finding a corresponding universal gauge of $\mathbf{G}$. This in turn prompts the natural next step to find a concrete methodology with a sound physical interpretation by means of which we can define a functorial gauge for an arbitrary covariant functor $\mathbf{F}: \mathfrak{C} \rightarrow \mathfrak{L}$ or contravariant functor $\mathbf{H}: \mathfrak{C}^{o p} \rightarrow \mathfrak{L}$.

A starting point to resolve this problem focusses on the observation that given any category $\mathfrak{A}$, the morphisms in $\mathfrak{A}$, or equivalently the morphisms in $\mathfrak{A}^{o p}$, can be defined as the elements in the values of the Hom $_{\mathfrak{A}}$-bifunctor:

$$
\mathbf{H o m}_{\mathfrak{A}}=\mathbf{y}(-,-)=\mathbf{y}_{(\mathcal{B})}^{(\mathcal{A})}=\mathfrak{A}^{o p} \times \mathfrak{A} \rightarrow \text { Sets }
$$

from the product category $\mathfrak{A}^{o p} \times \mathfrak{A}$ to the category of sets. This is because $\mathfrak{A}$ and $\mathfrak{A}^{o p}$ are considered to have the same objects and reversed morphisms. The $H_{\mathfrak{A}}$-bifunctor operates in such a way that by fixing an object $\mathcal{B}$ in $\mathfrak{A}^{o p}$ :

$$
\mathbf{y}_{\mathcal{B}}: \mathfrak{A} \rightarrow \text { Sets }
$$

is the covariant representable functor, or representable copresheaf of sets, represented by $\mathcal{B}$. Correspondingly by fixing an object $\mathcal{A}$ in $\mathfrak{A}$ :

$$
\mathbf{y}^{\mathcal{A}}: \mathfrak{A}^{o p} \rightarrow \text { Sets }
$$

is the contravariant representable functor, or representable presheaf of sets, represented by $\mathcal{A}$. It is also instructive to keep in mind that $\mathbf{y}^{\mathcal{A}}$ defines a representable left action of $\mathfrak{A}^{o p}$ on a set which is partitioned into sorts parameterized by the objects of $\mathfrak{A}^{o p}$, whereas $\mathbf{y}_{\mathcal{B}}$ defines a representable left action of $\mathfrak{A}$ on a set which is partitioned into sorts parameterized by the objects of $\mathfrak{A}$.

Now we may consider oppositely directing functors:

$$
\begin{aligned}
& \overrightarrow{\mathbf{T}}: \mathfrak{A}^{o p} \rightarrow \mathfrak{A} \\
& \overline{\mathbf{T}}: \mathfrak{A} \rightarrow \mathfrak{A}^{o p}
\end{aligned}
$$


which are tautological on objects and arrows-reversing. This being so, it is possible to rephrase the representability property of the $\mathrm{Hom}_{\mathfrak{A}}$ bifunctor with respect to fixing one of its arguments as follows:

i The bifunctor $\mathbf{y}(-,-)$ is representable in $\mathfrak{A}$ because for each $\mathfrak{A}^{o p}$-object $\mathcal{B}$ there exists a (tautological) $\mathfrak{A}$-object $\overrightarrow{\mathbf{T}}(\mathcal{B})$, such that $\mathbf{y}(\mathcal{B},-)$ is representable in $\mathfrak{A}$, that is:

$$
\operatorname{Hom}_{\mathfrak{A}}(\overrightarrow{\mathbf{T}}(\mathcal{B}), \mathcal{A}) \cong \mathbf{y}(\mathcal{B}, \mathcal{A})
$$

which is natural in the argument $\mathcal{A}$ in $\mathfrak{A}$.

ii The bifunctor $\mathbf{y}(-,-)$ is representable in $\mathfrak{A}^{o p}$ because for each $\mathfrak{A}$-object $\mathcal{A}$ there exists a (tautological) $\mathfrak{A}^{o p}$-object $\overline{\mathbf{T}}(\mathcal{A})$, such that $\mathbf{y}(-, \mathcal{A})$ is representable in $\mathfrak{A}^{o p}$, that is:

$$
\operatorname{Hom}_{\mathfrak{A}^{o p}}(\mathcal{B}, \overline{\mathbf{T}}(\mathcal{A})) \cong \mathbf{y}(\mathcal{B}, \mathcal{A})
$$

which is natural in the argument $\mathcal{B}$ in $\mathfrak{A}^{o p}$.

Thus, by combining the above, the representability of the bifunctor $\mathbf{y}(-,-)$ with respect to the categories $\mathfrak{A}$ and $\mathfrak{A}^{o p}$, or equivalently the birepresentability of the bifunctor $\mathbf{y}(-,-)$ with respect to $\mathfrak{A}$ and $\mathfrak{A}^{\text {op }}$ is expressed as follows:

$$
\operatorname{Hom}_{\mathfrak{A}}(\overrightarrow{\mathbf{T}}(\mathcal{B}), \mathcal{A}) \cong \mathbf{y}(\mathcal{B}, \mathcal{A}) \cong \operatorname{Hom}_{\mathfrak{A}^{o p}}(\mathcal{B}, \overline{\mathbf{T}}(\mathcal{A}))
$$

which is natural in both the arguments $\mathcal{B}$ in $\mathfrak{A}^{o p}$ and $\mathcal{A}$ in $\mathfrak{A}$.

Although the above is based on tautological functors, it contains the seeds for a powerful generalization in the case of general functors between categories pointing in opposite directions. This generalization is exactly the crucial step needed in order to tackle the problem of defining a functorial gauge (functorial reference frame) for an arbitrary covariant functor $\mathbf{F}: \mathfrak{C} \rightarrow \mathfrak{L}$ or contravariant functor $\mathbf{H}: \mathfrak{C}^{o p} \rightarrow \mathfrak{L}$. Since the distinction between the two cases can be handled trivially by means of tautological functors, from here on we formulate the solution to the problem posed above by referring to an arbitrary functor $\mathbf{F}: \mathfrak{C}^{o p} \rightarrow \mathfrak{L}$.

Before proceeding it is instructive to observe that in the above series of natural isomorphisms, the first term refers to morphisms taken 
in $\mathfrak{A}$ and the last term refers to morphisms taken in $\mathfrak{A}^{o p}$. Now, the middle term can refer either to morphisms in $\mathfrak{A}$ or to morphisms in $\mathfrak{A}^{o p}$ because of the tautological nature of the objects in opposite categories. Beyond this, if we temporarily overlook this tautology, it is particularly interesting to consider an element of $\mathbf{y}(\mathcal{B}, \mathcal{A})$ as a signaltransmitter from $\mathcal{B}$ in $\mathfrak{A}^{o p}$ to $\mathcal{A}$ in $\mathfrak{A}$, or equivalently, as a signalreceiver in the opposite direction.

Note that, if we overlook again the object tautology of these categories, a signal-transmitter/receiver is not a morphism within any of the categories $\mathfrak{A}^{o p}, \mathfrak{A}$, since it transfers a signal from some object in $\mathfrak{A}^{o p}$ to some object in $\mathfrak{A}$. Notwithstanding this fact, a signaltransmitter/receiver between objects in different categories can be made into a morphism in a new category, namely in the product category $\mathfrak{A}^{o p} \times \mathfrak{A}$ by considering the embeddings $\mathfrak{A}^{o p} \infty \mathfrak{A}^{o p} \times \mathfrak{A}$ and $\mathfrak{A} \infty \mathfrak{A}^{o p} \times \mathfrak{A}$.

The important fact is that the set of signal-transmitter/receivers for any objects $\mathcal{B}$ in $\mathfrak{A}^{o p}$ and $\mathcal{A}$ in $\mathfrak{A}$ can be represented as morphisms within both the categories in question, $\mathfrak{A}$ and $\mathfrak{A}^{o p}$, using the oppositely directing functors $\overrightarrow{\mathrm{T}}, \overline{\mathrm{T}}$, by means of the first and the last term in the series of isomorphisms. Moreover, since these isomorphisms are natural, the translation process effectuating representability in both directions becomes possible because there exist functorial gauges for both $\mathbf{y}_{\mathcal{B}}$ and $\mathbf{y}^{\mathcal{A}}$, or equivalently, universal gauges $\left(\mathcal{B}, i d_{B}\right),\left(\mathcal{A}, i d_{A}\right)$ of $\mathbf{y}_{\mathcal{B}}, \mathbf{y}^{\mathcal{A}}$ correspondingly.

By analogia, it is almost straightforward to proceed in the general case of a bifunctor:

$$
\wedge^{(-,-)}: \mathfrak{C}^{o p} \times \mathfrak{L} \rightarrow \text { Sets }
$$

from the product category $\mathfrak{C}^{o p} \times \mathfrak{L}$ to the category of sets. The elements in the values of $\wedge(-,-)$ are considered as signal-transmitter/receivers between objects of $\mathfrak{C}^{o p}, \mathfrak{L}$ correspondingly. It is important to notice the directionality build into the process of signal transfer, by which we mean that it is understood as a transfer from an object of $\mathfrak{C}^{o p}$ to an object of $\mathfrak{L}$. Again, a signal-transmitter/receiver between objects of $\mathfrak{C}^{o p}$, $\mathfrak{L}$ correspondingly, can be made into a morphism in a new category, namely in the product category $\mathfrak{C}^{o p} \times \mathfrak{L}$ by considering the embeddings $\mathfrak{C}^{o p} \infty \mathfrak{C}^{o p} \times \mathfrak{L}$ and $\mathfrak{L} \infty \mathfrak{C}^{o p} \times \mathfrak{L}$. 
We say that the bifunctor: $\wedge^{(-,-)}: \mathfrak{C}^{o p} \times \mathfrak{L} \rightarrow$ Sets is birepresentable, i.e. it is representable within both the categories $\mathfrak{C}^{o p}, \mathfrak{L}$, if and only if there exist two functors pointing into opposite directions, that is:

$$
\begin{aligned}
& \overrightarrow{\mathbf{A}}: \mathfrak{C}^{o p} \rightarrow \mathfrak{L} \\
& \overline{\mathbf{A}}: \mathfrak{L} \rightarrow \mathfrak{C}^{o p}
\end{aligned}
$$

and a series of isomorphisms:

$$
\operatorname{Hom}_{\mathfrak{L}}(\overrightarrow{\mathbf{A}}(\mathcal{C}), \mathcal{L}) \cong \wedge(\mathcal{C}, \mathcal{L}) \cong \operatorname{Hom}_{\mathfrak{C}^{o p}}(\mathcal{C}, \overline{\mathbf{A}}(\mathcal{L}))
$$

which is natural in both the arguments $\mathcal{C}$ in $\mathfrak{C}^{o p}$ and $\mathcal{L}$ in $\mathfrak{L}$.

Equivalently, we state that the bifunctor: $\wedge^{(-,-)}: \mathfrak{C}^{o p} \times \mathfrak{L} \rightarrow$ Sets is birepresentable, if and only if there exist two functors $\overrightarrow{\mathbf{A}}: \mathfrak{C}^{o p} \rightarrow \mathfrak{L}$, $\overline{\mathbf{A}}: \mathfrak{L} \rightarrow \mathfrak{C}^{o p}$ together with natural isomorphisms of bifunctors:

$$
\operatorname{Hom}_{\mathfrak{L}}(\overrightarrow{\mathbf{A}}(-),-) \cong \wedge(-,-) \cong \operatorname{Hom}_{\mathfrak{e}^{o p}}(-, \overline{\mathbf{A}}(-))
$$

In this case, we say that the two oppositely pointing functors $\overrightarrow{\mathbf{A}}, \overleftarrow{\mathbf{A}}$ form a categorical adjunction, which is induced by the requirement of

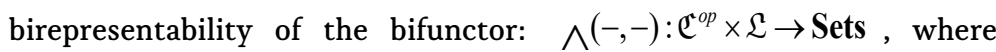
$\overrightarrow{\mathbf{A}}: \mathfrak{C}^{o p} \rightarrow \mathfrak{L}$ is the left adjoint functor of the adjunction, and symmetrically, $\overline{\mathbf{A}}: \mathfrak{L} \rightarrow \mathfrak{C}^{o p}$ is the right adjoint functor of the adjunction. The notion of a categorical adjunction was conceived and formulated by Daniel Kan.

If we ignore the middle term in the above series, we obtain the natural isomorphism of the Hom-bifunctors:

$$
\Psi: \operatorname{Hom}_{\mathfrak{L}}(\overrightarrow{\mathbf{A}}(-),-) \cong \operatorname{Hom}_{\mathfrak{c}^{o p}}(-, \overline{\mathbf{A}}(-))
$$

where, the bifunctors $\operatorname{Hom}_{\mathfrak{L}}(\overrightarrow{\mathbf{A}}(-),-), \operatorname{Hom}_{\mathfrak{C}^{o p}}(-, \overline{\mathbf{A}}(-))$ are obtained as follows: 


$$
\begin{aligned}
& \mathfrak{C}^{o p} \times \mathfrak{L} \stackrel{\overrightarrow{\mathrm{A}} \times \mathbf{i d} \mathbf{L}_{\mathfrak{L}}}{\longrightarrow} \quad \mathfrak{L}^{o p} \times \mathfrak{L} \stackrel{\text { Hom }_{\mathfrak{L}}}{\longrightarrow \text { Sets }} \\
& \mathfrak{C}^{o p} \times \mathfrak{L} \quad \stackrel{\operatorname{id}_{\mathfrak{C}^{o p}} \times \overleftarrow{\mathrm{A}}}{\longrightarrow} \quad \mathfrak{C}^{o p} \times \mathfrak{C} \stackrel{\text { Hom }_{\mathfrak{C}^{o p}}^{\longrightarrow} \text { Sets }}{ }
\end{aligned}
$$

Equivalently, we obtain the isomorphism:

$$
\Psi_{\mathcal{C}, \mathcal{L}}: \operatorname{Hom}_{\mathfrak{L}}(\overrightarrow{\mathbf{A}}(\mathcal{C}), \mathcal{L}) \cong \mathbf{H o m}_{\mathfrak{C}^{o p}}(\mathcal{C}, \overline{\mathbf{A}}(\mathcal{L}))
$$

which is natural in both the arguments $\mathcal{C}$ in $\mathfrak{C}^{o p}$ and $\mathcal{L}$ in $\mathfrak{L}$. The morphisms in $\mathfrak{L}, \mathfrak{C}^{o p}$ related to each other by the above isomorphism are called adjoint transposes. Hence, if we consider a morphism $h: \overrightarrow{\mathbf{A}}(\mathcal{C}) \rightarrow \mathcal{L}$ in $\mathfrak{L}$, we find by virtue of the adjunction isomorphism, it has an adjoint transpose morphism in $\mathfrak{C}^{o p}$, namely:

$$
\Psi_{\mathcal{C}, \mathcal{L}}(h)=h^{*}: \mathcal{C} \rightarrow \overline{\mathbf{A}}(\mathcal{L})
$$

In dual correlation, if we consider a morphism $g: \mathcal{C} \rightarrow \overline{\mathbf{A}}(\mathcal{L})$ in $\mathfrak{C}^{o p}$, we again find by virtue of the adjunction isomorphism, it has an adjoint transpose morphism in $\mathfrak{L}$, namely:

$$
\Psi_{\mathcal{C}, \mathcal{L}}^{-1}(g)=g^{*}: \overrightarrow{\mathbf{A}}(\mathcal{C}) \rightarrow \mathcal{L}
$$

Now, we may consider the identity morphism at $\overrightarrow{\mathbf{A}}(\mathcal{C})$ in $\mathfrak{L}$, that is $i d_{\overrightarrow{\mathbf{A}}(\mathcal{C})}$ belonging to the set $\operatorname{Hom}_{\mathfrak{L}}(\overrightarrow{\mathbf{A}}(\mathcal{C}), \overrightarrow{\mathbf{A}}(\mathcal{C}))$. The adjoint transpose of $i d_{\overrightarrow{\mathbf{A}}(\mathcal{C})}$ is called the unit morphism at $\mathcal{C}$, that is:

$$
\Psi_{\mathcal{C}, \overline{\mathbf{A}}(\mathcal{C})}\left(i d_{\overline{\mathbf{A}}(\mathcal{C})}\right)=\left(i d_{\overline{\mathbf{A}}(\mathcal{C})}\right)^{*}=\eta_{\mathcal{C}}: \mathcal{C} \rightarrow \overline{\mathbf{A}}(\overrightarrow{\mathbf{A}}(\mathcal{C}))
$$

belonging to the set $\operatorname{Hom}_{\mathfrak{c}^{o p}}(\mathcal{C}, \overline{\mathbf{A}}(\overrightarrow{\mathbf{A}}(\mathcal{C})))$. Since the above is natural on the argument $\mathcal{C}$ in $\mathfrak{C}^{o p}$, we obtain a natural transformation of the identity functor on $\mathfrak{C}^{o p}$, called the unit natural transformation of the adjunction: 


$$
\eta: \mathbf{i d}_{\mathfrak{C}^{\mathbf{o p}}} \rightarrow \overrightarrow{\mathbf{A}} \overrightarrow{\mathbf{A}}
$$

As a further dual correlation, we may consider the identity morphism at $\overline{\mathbf{A}}(\mathcal{L})$ in $\mathfrak{C}^{o p}$, that is $i d_{\overline{\mathbf{A}}(\mathcal{L})}$ belonging to the set $\operatorname{Hom}_{\mathfrak{C}^{o p}}(\overline{\mathbf{A}}(\mathcal{L}), \overline{\mathbf{A}}(\mathcal{L}))$. The adjoint transpose of $i d_{\overline{\mathbf{A}}(\mathcal{L})}$ is called the counit morphism at $\mathcal{L}$, that is:

$$
\Psi_{\overline{\mathbf{A}}(\mathcal{L}), \mathcal{L}}^{-1}\left(i d_{\overline{\mathbf{A}}(\mathcal{L})}\right)=\left(i d_{\overline{\mathbf{A}}(\mathcal{L})}\right)^{*}=\varepsilon_{\mathcal{L}}: \overrightarrow{\mathbf{A}}(\overline{\mathbf{A}}(\mathcal{L})) \rightarrow \mathcal{L}
$$

belonging to the set $\operatorname{Hom}_{\mathfrak{L}}(\overrightarrow{\mathbf{A}}(\overleftarrow{\mathbf{A}}(\mathcal{L})), \mathcal{L})$. Since the above is natural on the argument $\mathcal{L}$ in $\mathfrak{L}$, we obtain a natural transformation of the identity functor on $\mathfrak{L}$, called the counit natural transformation of the adjunction:

$$
\varepsilon: \overrightarrow{\mathbf{A A}} \rightarrow \mathbf{i d}_{\mathfrak{L}}
$$

From the unit and counit natural transformations of the adjunction we obtain the following identities, called triangular identities:

$$
\begin{aligned}
& \overrightarrow{\mathbf{A}} \stackrel{\overrightarrow{\mathbf{A}} \eta}{\longrightarrow} \overrightarrow{\mathbf{A}} \overleftarrow{\mathbf{A}} \overrightarrow{\mathbf{A}} \stackrel{\varepsilon \overrightarrow{\mathbf{A}}}{\longrightarrow} \overrightarrow{\mathbf{A}} \\
& \overleftarrow{\mathbf{A}} \stackrel{\eta \overleftarrow{\mathbf{A}}}{\longrightarrow} \overleftarrow{\mathbf{A}} \overrightarrow{\mathbf{A}} \overleftarrow{\mathbf{A}} \stackrel{\overleftarrow{\mathbf{A}} \varepsilon}{\longrightarrow} \overleftarrow{\mathbf{A}}
\end{aligned}
$$

The above identities may be written equivalently in equation form as follows:

$$
\begin{aligned}
& i d_{\overline{\mathbf{A}}}=\varepsilon \overrightarrow{\mathbf{A}} \circ \overrightarrow{\mathbf{A}} \eta \\
& i d_{\overline{\mathbf{A}}}=\overleftarrow{\mathbf{A}} \varepsilon \circ \eta \overline{\mathbf{A}}
\end{aligned}
$$

where, $i d_{\overline{\mathbf{A}}}, i d_{\overline{\mathbf{A}}}$ denote the identity natural transformations on the functors $\overrightarrow{\mathbf{A}}, \overline{\mathbf{A}}$ respectively. We conclude that the categorical adjunction being formed by $\overrightarrow{\mathbf{A}}, \overline{\mathbf{A}}$ can be represented equivalently in terms of the unit and counit natural transformations obeying the triangular identities. 
Next, we observe that if we consider a morphism $h: \overrightarrow{\mathbf{A}}(\mathcal{C}) \rightarrow \mathcal{L}$ in $\mathfrak{L}$, by virtue of the unit natural transformation, its adjoint transpose morphism in $\mathfrak{C}^{o p}$, namely:

$$
\Psi_{\mathcal{C}, \mathcal{L}}(h)=h^{*}: \mathcal{C} \rightarrow \overline{\mathbf{A}}(\mathcal{L})
$$

factors uniquely via the unit morphism at $\mathcal{C}$ as follows:

$$
\mathcal{C} \stackrel{\eta_{\mathcal{C}}}{\longrightarrow} \overleftarrow{\mathbf{A}} \overrightarrow{\mathbf{A}}(\mathcal{C}) \stackrel{\overleftarrow{\mathbf{A}} h}{\longrightarrow} \overleftarrow{\mathbf{A}}(\mathcal{L})
$$

or, more explicitly:

$$
\Psi_{\mathcal{C}, \mathcal{L}}(h)=h^{*}=\overline{\mathbf{A}}(h) \circ \eta_{\mathcal{C}}: \mathcal{C} \rightarrow \overline{\mathbf{A}}(\mathcal{L})
$$

Thus, the pair $\left(\overrightarrow{\mathbf{A}}(\mathcal{C}), \eta_{\mathcal{C}}\right)$ constitutes a universal gauge for the functor:

$$
\operatorname{Hom}_{\mathfrak{c}^{o p}}(\mathcal{C}, \overline{\mathbf{A}}(-))=\mathbf{y}_{\mathcal{C}} \circ \overline{\mathbf{A}}: \mathfrak{L} \rightarrow \text { Sets }
$$

Equivalently stated, the pair $\left(\overrightarrow{\mathbf{A}}(\mathcal{C}), \eta_{\mathcal{C}}\right)$ is an initial object in the category of elements of the Sets-valued functor $\mathbf{y}_{\mathcal{C}} \circ \overleftarrow{\mathbf{A}}: \mathfrak{L} \rightarrow$ Sets

In dual correlation, if we consider a morphism $g: \mathcal{C} \rightarrow \overline{\mathbf{A}}(\mathcal{L})$ in $\mathfrak{C}^{o p}$, by virtue of the counit natural transformation, its adjoint transpose morphism in $\mathfrak{L}$, namely:

$$
\Psi_{\mathcal{C}, \mathcal{L}}^{-1}(g)=g^{*}: \overrightarrow{\mathbf{A}}(\mathcal{C}) \rightarrow \mathcal{L}
$$

factors uniquely via the counit morphism at $\mathcal{L}$ as follows:

$$
\overrightarrow{\mathbf{A}}(\mathcal{C}) \stackrel{\overrightarrow{\mathbf{A}} g}{\longrightarrow} \overrightarrow{\mathbf{A}} \overleftarrow{\mathbf{A}}(\mathcal{L}) \stackrel{\varepsilon_{\mathcal{L}}}{\longrightarrow} \mathcal{L}
$$

or, more explicitly:

$$
\Psi^{-1}{ }_{\mathcal{C}, \mathcal{L}}(g)=g^{*}=\varepsilon_{\mathcal{L}} \circ \overrightarrow{\mathbf{A}}(g): \overrightarrow{\mathbf{A}}(\mathcal{C}) \rightarrow \mathcal{L}
$$


Thus, the pair $\left(\overleftarrow{\mathbf{A}}(\mathcal{L}), \varepsilon_{\mathcal{L}}\right)$ constitutes a universal gauge for the functor:

$$
\operatorname{Hom}_{\mathfrak{L}}(\overrightarrow{\mathbf{A}}(-), \mathcal{L})=\mathbf{y}^{\mathcal{L}} \circ \overrightarrow{\mathbf{A}}: \mathfrak{C}^{o p} \rightarrow \text { Sets }
$$

This is to say that the pair $\left(\overleftarrow{\mathbf{A}}(\mathcal{L}), \varepsilon_{\mathcal{L}}\right)$ is a terminal object in the category of elements of the Sets-valued functor $\mathbf{y}^{\mathcal{L}} \circ \overrightarrow{\mathbf{A}}: \mathfrak{C}^{o p} \rightarrow$ Sets.

Let us focus again on the bifunctor: $\wedge^{(-,-)}: \mathfrak{C}^{o p} \times \mathfrak{L} \rightarrow$ Sets. We see immediately that if the bifunctor $\wedge^{(-,-)}$is birepresentable, then the set of signal-transmitter/receivers for any objects $\mathcal{C}$ in $\mathfrak{C}^{o p}$ and $\mathcal{L}$ in $\mathfrak{L}$ can be represented as morphisms within both the involved categories $\mathfrak{L}, \mathfrak{C}^{o p}$, using the left and right adjoint functors $\overrightarrow{\mathbf{A}}, \overline{\mathbf{A}}$ of the induced adjunction, by means of the first and the last term in the series of isomorphisms. Moreover, since these isomorphisms are natural, the translation process effectuating representability in both directions becomes possible, since there exist functorial gauges for both the functors:

$$
\begin{gathered}
\wedge^{(\mathcal{C},-)}: \mathfrak{L} \rightarrow \text { Sets } \\
\wedge^{(-, \mathcal{L}): \mathfrak{C}^{o p} \rightarrow \text { Sets }}
\end{gathered}
$$

More concretely, for each $\mathcal{C}$ in $\mathfrak{C}^{o p}$, there exists a representing element $\overrightarrow{\mathbf{A}}(\mathcal{C})$ in $\mathfrak{L}$, such that:

$$
\Theta_{\mathcal{C}, \mathcal{L}}: \mathbf{H o m}_{\mathcal{L}}(\overrightarrow{\mathbf{A}}(\mathcal{C}), \mathcal{L}) \cong \wedge(\mathcal{C}, \mathcal{L})
$$

which is natural in $\mathcal{L}$. For $\mathcal{L}=\overrightarrow{\mathbf{A}}(\mathcal{C})$ we obtain:

$$
\Theta_{\mathcal{C}, \overline{\mathbf{A}}(\mathcal{C})}: \mathbf{H o m}_{\mathfrak{L}}(\overrightarrow{\mathbf{A}}(\mathcal{C}), \overrightarrow{\mathbf{A}}(\mathcal{C})) \cong \wedge(\mathcal{C}, \overrightarrow{\mathbf{A}}(\mathcal{C}))
$$

Now, we may consider the identity morphism at $\overrightarrow{\mathbf{A}}(\mathcal{C})$ in $\mathfrak{L}$, that is, $i d_{\overrightarrow{\mathbf{A}}(\mathcal{C})}$ belonging to the set $\operatorname{Hom}_{\mathfrak{L}}(\overrightarrow{\mathbf{A}}(\mathcal{C}), \overrightarrow{\mathbf{A}}(\mathcal{C}))$. The image of $i d_{\overrightarrow{\mathbf{A}}(\mathcal{C})}$ in

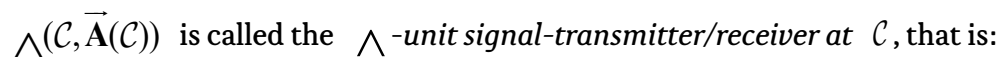

$$
\Theta_{\mathcal{C}, \overline{\mathbf{A}}(\mathcal{C})}\left(i d_{\overline{\mathbf{A}}(\mathcal{C})}\right)=\delta_{\mathcal{C}}: \mathcal{C} \rightarrow \overrightarrow{\mathbf{A}}(\mathcal{C})
$$


belonging to the set $\wedge(\mathcal{C}, \overrightarrow{\mathbf{A}}(\mathcal{C}))$. Since the above is natural on the argument $\mathcal{C}$ in $\mathfrak{C}^{o p}$, we obtain a natural transformation of the identity

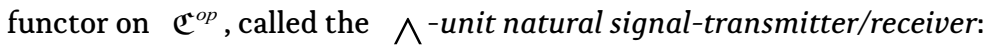

$$
\delta: \mathbf{i d}_{\mathfrak{C}^{\text {op }}} \rightarrow \overrightarrow{\mathbf{A}}
$$

We conclude that the pair $\left(\overrightarrow{\mathbf{A}}(\mathcal{C}), \delta_{\mathcal{C}}\right)$ constitutes a universal gauge for the functor:

$$
\wedge(\mathcal{C},-): \mathfrak{L} \rightarrow \text { Sets }
$$

Equivalently stated, the pair $\left(\overrightarrow{\mathbf{A}}(\mathcal{C}), \delta_{\mathcal{C}}\right)$ is an initial object in the category of elements of the Sets-valued functor $\wedge(\mathcal{C},-)$.

Similarly, for each $\mathcal{L}$ in $\mathfrak{L}$, there exists a representing element $\overline{\mathbf{A}}(\mathcal{L})$ in $\mathfrak{C}^{o p}$, such that:

$$
\Phi_{\mathcal{C}, \mathcal{L}}: \wedge(\mathcal{C}, \mathcal{L}) \cong \mathbf{H o m}_{\mathfrak{e}^{o p}}(\mathcal{C}, \overline{\mathbf{A}}(\mathcal{L}))
$$

which is natural in $\mathcal{C}$. For $\mathcal{C}=\overline{\mathbf{A}}(\mathcal{L})$ we obtain:

$$
\Phi_{\overline{\mathbf{A}}(\mathcal{L}), \mathcal{L}}: \wedge(\overline{\mathbf{A}}(\mathcal{L}), \mathcal{L}) \cong \mathbf{H o m}_{\mathfrak{c}^{o p}}(\overline{\mathbf{A}}(\mathcal{L}), \overline{\mathbf{A}}(\mathcal{L}))
$$

Now, we may consider the identity morphism at $\overline{\mathbf{A}}(\mathcal{L})$ in $\mathfrak{C}^{o p}$, that is $i d_{\overline{\mathbf{A}}(\mathcal{L})}$ belonging to the set $\mathbf{H o m}_{\mathbb{C}^{o p}}(\overline{\mathbf{A}}(\mathcal{L}), \overline{\mathbf{A}}(\mathcal{L}))$. The inverse image of $i d_{\overline{\mathbf{A}}(\mathcal{L})}$ in $\wedge(\overline{\mathbf{A}}(\mathcal{L}), \mathcal{L})$ is called the $\wedge$-counit signal transmitter/receiver at $\mathcal{L}$, that is:

$$
\Phi_{\overline{\mathbf{A}}(\mathcal{L}), \mathcal{L}}^{-1}\left(i d_{\overline{\mathbf{A}}(\mathcal{L})}\right)=\underline{U}_{\mathcal{L}}: \overline{\mathbf{A}}(\mathcal{L}) \rightarrow \mathcal{L}
$$

belonging to the set $\wedge(\overline{\mathbf{A}}(\mathcal{L}), \mathcal{L})$. Since the above is natural on the argument $\mathcal{L}$ in $\mathfrak{L}$, we obtain a natural transformation of the identity

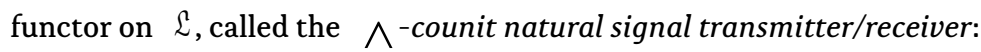

$$
\text { Ú: } \overline{\mathbf{A}} \rightarrow \mathbf{i d}_{\mathfrak{L}}
$$


We conclude that the pair $\left(\overleftarrow{\mathbf{A}}(\mathcal{L}), \bigcup_{\mathcal{L}}\right)$ constitutes a universal gauge for the functor:

$$
\wedge(-, \mathcal{L}): \mathfrak{C}^{o p} \rightarrow \text { Sets }
$$

which again provides that the pair $\left(\overline{\mathbf{A}}(\mathcal{L}), \bigcup_{\mathcal{L}}\right)$ is a terminal object in the

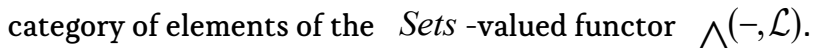

Therefore, if we consider a signal transmitter/receiver $\chi \in$ $\wedge(\mathcal{C}, \mathcal{L})$, the corresponding morphisms, induced by the birepresentability of the bifunctor:

$$
\wedge^{(-,-)}: \mathfrak{C}^{o p} \times \mathfrak{L} \rightarrow \text { Sets }
$$

within $\mathfrak{C}^{o p}, \mathfrak{L}$ respectively, that is:

$$
\begin{array}{r}
g(\chi): \mathcal{C} \rightarrow \overleftarrow{\mathbf{A}}(\mathcal{L}) \\
h(\chi): \overrightarrow{\mathbf{A}}(\mathcal{C}) \rightarrow \mathcal{L}
\end{array}
$$

play the role of conjugates with respect to the signal transmitter/receiver $\chi$.

As a consequence, if $\chi$ is the $\wedge$-unit signaltransmitter/receiver at $\mathcal{C}$, namely $\chi=\delta_{\mathcal{C}}: \mathcal{C} \rightarrow \overrightarrow{\mathbf{A}}(\mathcal{C})$, then:

$$
\begin{gathered}
g\left(\delta_{\mathcal{C}}\right)=\eta_{\mathcal{C}}: \mathcal{C} \rightarrow \overline{\mathbf{A}}(\overrightarrow{\mathbf{A}}(\mathcal{C})) \\
h\left(\delta_{\mathcal{C}}\right)=i d_{\overline{\mathbf{A}}(\mathcal{C})}: \overrightarrow{\mathbf{A}}(\mathcal{C}) \rightarrow \overrightarrow{\mathbf{A}}(\mathcal{C})
\end{gathered}
$$

are conjugates with respect to the $\wedge$-unit signal-transmitter/receiver at $\mathcal{C}$. Since the above is natural on the argument $\mathcal{C}$ in $\mathfrak{C}^{o p}$, we conclude that:

$$
\begin{gathered}
g(\delta)=\eta: \mathbf{i d}_{\mathfrak{c}^{\text {op }}} \rightarrow \overrightarrow{\mathbf{A}} \overrightarrow{\mathbf{A}} \\
h(\delta)=i d_{\overrightarrow{\mathbf{A}}}: \overrightarrow{\mathbf{A}} \rightarrow \overrightarrow{\mathbf{A}}
\end{gathered}
$$

Which is to say that the unit natural transformation of the adjunction $\eta$, and the $\overrightarrow{\mathbf{A}}$-identity natural transformation $i d_{\overrightarrow{\mathbf{A}}}$ are functorial 
conjugates with respect to the $\wedge$-unit natural signaltransmitter/receiver $\delta: \mathbf{i d}_{\mathfrak{C}^{\text {op }}} \rightarrow \overrightarrow{\mathbf{A}}$. Similarly, we conclude that:

$$
\begin{aligned}
g\left(U^{\prime}\right) & =\varepsilon: \overrightarrow{\mathbf{A}} \overleftarrow{\mathbf{A}} \rightarrow \mathbf{i d}_{\mathfrak{L}} \\
h\left(U^{\prime}\right) & =i d_{\overline{\mathbf{A}}}: \overline{\mathbf{A}} \rightarrow \overleftarrow{\mathbf{A}}
\end{aligned}
$$

meaning that, the counit natural transformation of the adjunction $\varepsilon$, and the $\overline{\mathbf{A}}$-identity natural transformation $i d_{\overline{\mathbf{A}}}$ are functorial conjugates with respect to the $\wedge$-counit natural signal-transmitter/receiver Ú: $\overline{\mathbf{A}} \rightarrow \mathbf{i d}_{\mathfrak{L}}$.

Furthermore, if we consider an arbitrary signal transmitter/receiver $\chi \in \wedge(\mathcal{C}, \mathcal{L})$, we obtain the following factorizations:

$$
\begin{aligned}
& \chi=h(\chi) \circ \delta_{\mathcal{C}} \\
& \chi=U_{\mathcal{L}} \circ g(\chi)
\end{aligned}
$$

presented equivalently, as follows:

$$
h(\chi) \circ \delta_{\mathcal{C}}=\chi=U_{\mathcal{L}} \circ g(\chi)
$$

where, $h(\chi), g(\chi)$ are conjugates with respect to the signal transmitter/receiver $\chi$. Denoting the conjugate of $h(\chi)$, that is $g(\chi)$, by $g(\chi)=h^{*}(\chi)$, or equivalently, identifying the conjugate of $h(\chi)$ (with respect to the signal transmitter/receiver $\chi$ ) with the adjoint transpose of $h(\chi)$, we obtain:

$$
h(\chi) \circ \delta_{\mathcal{C}}=\chi=\hat{U}_{\mathcal{L}} \circ h^{*}(\chi)
$$

Now, we are ready to tackle the problem of defining a functorial gauge (functorial reference frame) for an arbitrary functor. We say that a functor $\mathbf{F}: \mathfrak{C}^{o p} \rightarrow \mathfrak{L}$ has a functorial gauge relative to $\mathfrak{L}$ if and only if the bifunctor: $\wedge^{(-,-)}: \mathfrak{C}^{o p} \times \mathfrak{L} \rightarrow$ Sets is representable within $\mathfrak{L}$, that is we have a natural isomorphism of bifunctors:

$$
\operatorname{Hom}_{\mathfrak{L}}(\mathbf{F}(-),-) \cong \wedge^{(-,-)}
$$


Equivalently, the functor $\mathbf{F}: \mathfrak{C}^{o p} \rightarrow \mathfrak{L}$ has a functorial gauge relative to $\mathfrak{L}$ if and only if the functor:

$$
\wedge^{(\mathcal{C},-): \mathfrak{L} \rightarrow \text { Sets }}
$$

is representable, that is for each $\mathcal{C}$ in $\mathfrak{C}^{o p}$, there exists a representing element $\mathbf{F}(\mathcal{C})$ in $\mathfrak{L}$, such that:

$$
\Theta_{\mathcal{C}, \mathcal{L}}: \operatorname{Hom}_{\mathfrak{L}}(\mathbf{F}(\mathcal{C}), \mathcal{L}) \cong \wedge(\mathcal{C}, \mathcal{L})
$$

which is natural in $\mathcal{L}$.

Equivalently, the functor $\mathbf{F}: \mathfrak{C}^{o p} \rightarrow \mathfrak{L}$ has a functorial gauge relatively to $\mathfrak{L}$ if and only if the pair $\left(\mathbf{F}(\mathcal{C}), \delta_{\mathcal{C}}: \mathcal{C} \rightarrow \mathbf{F}(\mathcal{C})\right)$ is a universal gauge for the functor $\wedge(\mathcal{C},-)$.

Symmetrically, we say that an oppositely pointing functor $\mathbf{G}: \mathfrak{L} \rightarrow \mathfrak{C}^{o p}$ has a functorial gauge relative to $\mathfrak{C}^{o p}$ if and only if the bifunctor: $\wedge^{(-,-)}: \mathfrak{C}^{o p} \times \mathfrak{L} \rightarrow$ Sets is representable within $\mathfrak{C}^{o p}$, that is, we have a natural isomorphism of bifunctors:

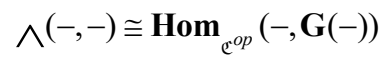

Equivalently, the functor $\mathbf{G}: \mathfrak{L} \rightarrow \mathfrak{C}^{o p}$ has a functorial gauge relative to $\mathfrak{C}^{o p}$ if and only if the functor:

$$
\wedge(-, \mathcal{L}): \mathfrak{C}^{o p} \rightarrow \text { Sets }
$$

is representable, that is for each $\mathcal{L}$ in $\mathfrak{L}$, there exists a representing element $\mathbf{G}(\mathcal{L})$ in $\mathfrak{C}^{o p}$, such that:

$$
\Phi_{\mathcal{C}, \mathcal{L}}: \wedge(\mathcal{C}, \mathcal{L}) \cong \mathbf{H o m}_{\mathfrak{C}^{o p}}(\mathcal{C}, \mathbf{G}(\mathcal{L}))
$$

which is natural in $\mathcal{C}$.

Equivalently, the functor $\mathbf{G}: \mathfrak{L} \rightarrow \mathfrak{C}^{o p}$ has a functorial gauge relative to $\mathfrak{C}^{o p}$ if and only if the pair $\left(\mathbf{G}(\mathcal{L}), \cup_{\mathcal{L}}: \mathbf{G}(\mathcal{L}) \rightarrow \mathcal{L}\right)$ is a universal gauge for the functor $\wedge(-, \mathcal{L})$.

We say that an arbitrary functor of the form $\mathbf{F}: \mathfrak{C}^{o p} \rightarrow \mathfrak{L}$ has a functorial gauge if and only if it has a right adjoint functor $\mathbf{G}: \mathfrak{L} \rightarrow \mathfrak{C}^{o p}$. 
Equivalently, the functor $\mathbf{F}: \mathfrak{C}^{o p} \rightarrow \mathfrak{L}$ has a functorial gauge if and only if each functor $\mathbf{y}^{\mathcal{L}} \circ \mathbf{F}$ is representable for each object $\mathcal{L}$ in $\mathfrak{L}$. If this is the case, a functor $\mathbf{G}: \mathfrak{L} \rightarrow \mathfrak{C}^{o p}$ right adjoint to $\mathbf{F}: \mathfrak{C}^{o p} \rightarrow \mathfrak{L}$ assigns to each $\mathcal{L}$ in $\mathfrak{L}$ an object $\mathbf{G}(\mathcal{L})$ in $\mathfrak{C}^{o p}$ representing the functor:

$$
\operatorname{Hom}_{\mathfrak{L}}(\mathbf{F}(-), \mathcal{L})=\mathbf{y}^{\mathcal{L}} \circ \mathbf{F}: \mathfrak{C}^{o p} \rightarrow \text { Sets }
$$

Moreover, any other functor right adjoint to $\mathrm{F}$ is naturally isomorphic with G.

Equivalently, the functor $\mathbf{F}: \mathfrak{C}^{o p} \rightarrow \mathfrak{L}$ has a functorial gauge if and only if there exists for each object $\mathcal{L}$ in $\mathfrak{L}$ an object $\mathbf{G}(\mathcal{L})$ in $\mathfrak{C}^{o p}$ and an arrow $\varepsilon_{\mathcal{L}}: \mathbf{F}(\mathbf{G}(\mathcal{L})) \rightarrow \mathcal{L}$, such that the pair $\left(\mathbf{G}(\mathcal{L}), \varepsilon_{\mathcal{L}}\right)$ constitutes a universal gauge for the functor $\mathbf{y}^{\mathcal{L}} \circ \mathbf{F}$.

Symmetrically, we say that an arbitrary functor of the form $\mathbf{G}: \mathfrak{L} \rightarrow \mathfrak{C}^{o p}$ has a functorial gauge if and only if it has a left adjoint functor $\mathbf{F}: \mathfrak{C}^{o p} \rightarrow \mathfrak{L}$.

Equivalently, the functor $\mathbf{G}: \mathfrak{L} \rightarrow \mathfrak{C}^{o p}$ has a functorial gauge if and only if each functor $\mathbf{y}_{\mathcal{C}} \circ \mathbf{G}$ is representable for each object $\mathcal{C}$ in $\mathfrak{C}^{o p}$. If this is the case, a functor $\mathbf{F}: \mathfrak{C}^{o p} \rightarrow \mathfrak{L}$ left adjoint to $\mathbf{G}: \mathfrak{L} \rightarrow \mathfrak{C}^{o p}$ assigns to each $\mathcal{C}$ in $\mathfrak{C}^{o p}$ an object $\mathbf{F}(\mathcal{C})$ in $\mathfrak{L}$ representing the functor:

$$
\mathbf{H o m}_{\mathfrak{C}^{o p}}(\mathcal{C}, \mathbf{G}(-))=\mathbf{y}_{\mathcal{C}} \circ \mathbf{G}: \mathfrak{L} \rightarrow \text { Sets }
$$

Moreover, any other functor left adjoint to $\mathbf{G}$ is naturally isomorphic with F.

Equivalently, the functor $\mathbf{G}: \mathfrak{L} \rightarrow \mathfrak{C}^{o p}$ has a functorial gauge if and only if there exists for each object $\mathcal{C}$ in $\mathfrak{C}^{o p}$ an object $\mathbf{F}(\mathcal{C})$ in $\mathfrak{L}$ and an arrow $\eta_{\mathcal{C}}: \mathcal{C} \rightarrow \mathbf{G}(\mathbf{F}(\mathcal{C}))$, such that the pair $\left(\mathbf{F}(\mathcal{C}), \eta_{\mathcal{C}}\right)$ constitutes a universal gauge for the functor $\mathbf{y}_{\mathcal{C}} \circ \mathbf{G}$.

Finally, we say that two oppositely pointing functors between the same categories, that is, $\mathbf{F}: \mathfrak{C}^{o p} \rightarrow \mathfrak{L}, \mathbf{G}: \mathfrak{L} \rightarrow \mathfrak{C}^{o p}$, have mutual functorial gauges with respect to each other if and only if the functor $\mathbf{F}$ is left adjoint to the functor $\mathbf{G}$ and the functor $\mathbf{G}$ is right adjoint to the functor $\mathbf{F}$, such that, the two oppositely pointing functors $\overrightarrow{\mathbf{A}}:=\mathbf{F}$, 
$\overline{\mathbf{A}}:=\mathbf{G}$ form a categorical adjunction, expressed by the natural isomorphism of the Hom-bifunctors:

$$
\Psi: \operatorname{Hom}_{\mathfrak{L}}(\overrightarrow{\mathbf{A}}(-),-) \cong \operatorname{Hom}_{\mathfrak{c}^{o p}}(-, \overline{\mathbf{A}}(-))
$$

Equivalently, $\overrightarrow{\mathbf{A}}, \overleftarrow{\mathbf{A}}$ have mutual functorial gauges with respect to each other if and only if the bifunctor $\wedge^{(-,-)}: \mathfrak{C}^{o p} \times \mathfrak{L} \rightarrow$ Sets is birepresentable, that is, we have a series of natural isomorphisms of bifunctors:

$$
\operatorname{Hom}_{\mathfrak{L}}(\overrightarrow{\mathbf{A}}(-),-) \cong \wedge(-,-) \cong \operatorname{Hom}_{\mathfrak{c}^{o p}}(-, \overline{\mathbf{A}}(-))
$$

When this is the case, the two oppositely pointing functors $\overrightarrow{\mathbf{A}}, \overleftarrow{\mathbf{A}}$ form a categorical adjunction, induced by the requirement of birepresentability of the bifunctor $\wedge^{(-,-)}$, where $\overrightarrow{\mathbf{A}}$ is the left adjoint functor of the adjunction, and symmetrically, $\overline{\mathbf{A}}$ is the right adjoint functor of the adjunction.

Equivalently, $\overrightarrow{\mathbf{A}}, \overline{\mathbf{A}}$ have mutual functorial gauges with respect to each other if and only if there exist a natural transformation of the identity functor on $\mathfrak{C}^{o p}$ (unit natural transformation):

$$
\eta: \mathbf{i d}_{\mathfrak{C}^{\text {op }}} \rightarrow \overleftrightarrow{\mathbf{A}} \overrightarrow{\mathbf{A}}
$$

as well as a natural transformation of the identity functor on $\mathfrak{L}$ (counit natural transformation):

$$
\varepsilon: \overrightarrow{\mathbf{A A}} \rightarrow \mathbf{i d}_{\mathfrak{L}}
$$

obeying the following identities:

$$
\begin{aligned}
& i d_{\overline{\mathbf{A}}}=\varepsilon \overrightarrow{\mathbf{A}} \circ \overrightarrow{\mathbf{A}} \eta \\
& i d_{\overline{\mathbf{A}}}=\overline{\mathbf{A}} \varepsilon \circ \eta \overline{\mathbf{A}}
\end{aligned}
$$

Equivalently, $\overrightarrow{\mathbf{A}}=\mathbf{F}, \overline{\mathbf{A}}=\mathbf{G}$ have mutual functorial gauges with respect to each other if and only if, each functor $\mathbf{y}^{\mathcal{L}} \circ \mathbf{F}$ is representable (via $\mathbf{G}$ ) for each object $\mathcal{L}$ in $\mathfrak{L}$, and each functor 
$\mathbf{y}_{\mathcal{C}} \circ \mathbf{G}$ is representable (via $\mathbf{F}$ ) for each object $\mathcal{C}$ in $\mathfrak{C}^{o p}$, that is, if and only if:

i There exists for each object $\mathcal{L}$ in $\mathfrak{L}$ an object $\mathbf{G}(\mathcal{L})$ in $\mathfrak{C}^{\text {op }}$ and an arrow $\varepsilon_{\mathcal{L}}: \mathbf{F}(\mathbf{G}(\mathcal{L})) \rightarrow \mathcal{L}$, such that the pair $\left(\mathbf{G}(\mathcal{L}), \varepsilon_{\mathcal{L}}\right)$ constitutes a universal gauge for the functor $\mathbf{y}^{\mathcal{L}} \circ \mathbf{F}$.

ii There exists for each object $\mathcal{C}$ in $\mathfrak{C}^{o p}$ an object $\mathbf{F}(\mathcal{C})$ in $\mathfrak{L}$ and an arrow $\eta_{\mathcal{C}}: \mathcal{C} \rightarrow \mathbf{G}(\mathbf{F}(\mathcal{C}))$, such that the pair $\left(\mathbf{F}(\mathcal{C}), \eta_{\mathcal{C}}\right)$ constitutes a universal gauge for the functor $\mathbf{y}_{\mathcal{C}} \circ \mathbf{G}$.

Equivalently, $\overrightarrow{\mathbf{A}}=\mathbf{F}, \quad \overline{\mathbf{A}}=\mathbf{G}$ have mutual functorial gauges with respect to each other if and only if:

i The functor $\wedge(\mathcal{C},-): \mathfrak{L} \rightarrow$ Sets is representable, such that the pair $\left(\mathbf{F}(\mathcal{C}), \delta_{\mathcal{C}}\right)$ is a universal gauge for the functor $\wedge(\mathcal{C},-)$.

ii The functor $\wedge(-, \mathcal{L}): \mathfrak{C}^{o p} \rightarrow$ Sets is representable, such that the pair $\left(\mathbf{G}(\mathcal{L}), \bigcup_{\mathcal{L}}\right)$ is a universal gauge for the functor $\wedge(-, \mathcal{L})$.

Let us now present some important consequences of the existence of mutual functorial gauges for two oppositely pointing functors between the same categories. As a simple illustration we may consider the diagonal functor:

$$
\Delta: \mathfrak{K} \rightarrow \mathfrak{K}^{\mathfrak{A}}
$$

For an object $\mathcal{K}$ in $\mathfrak{K}$, the functor $\Delta(\mathcal{K})$ is the constant $\mathcal{K}$-valued functor, which for any object $\mathcal{U}$ of a (locally) small category $\mathfrak{A}$ gives the constant value $\mathcal{K}$, and for any arrow $f$ of $\mathfrak{A}$ gives the identity arrow on $\mathcal{K}$. Furthermore, if we consider that $\mathfrak{K}$ is the category of Sets, then Sets $^{\mathfrak{A}}$ is the functor category of copresheaves (of sets), that is, the category of all covariant functors $\mathrm{P}$ from $\mathfrak{A}$ to Sets and all natural transformations between them, where $\mathfrak{A}$ is an indexing (locally) small category.

$$
\Delta: \text { Sets } \rightarrow \text { Sets }^{\mathfrak{A}}
$$




$$
\Delta(\mathcal{K}): \mathfrak{A} \rightarrow \text { Sets }
$$

Thus, $\Delta(\mathcal{K})$ is the constant $\mathcal{K}$-valued functor taking each object $\mathcal{U}$ in $\mathfrak{A}$ to the set $\mathcal{K}$, and each arrow $f: \mathcal{U} \rightarrow \mathcal{V}$ in $\mathfrak{A}$ to the identity morphism $i d_{\mathcal{K}}$. Now, if we consider the diagonal functor $\Delta$ as a functor of the above form $\mathrm{F}$, then it has a functorial gauge if and only if it has a right adjoint functor of the above form $\mathbf{G}$, denoted by:

$$
\text { O : } \text { Sets }^{\mathfrak{A}} \rightarrow \text { Sets }
$$

Moreover, the oppositely pointing functors $\Delta, \mathbf{O}$ have mutual functorial gauges with respect to each other if and only if $\Delta$ is left adjoint to $\mathbf{O}$ and $\mathbf{O}$ is right adjoint to $\Delta$, such that, the functors $\overrightarrow{\mathbf{A}}:=\Delta, \quad \overline{\mathbf{A}}:=\mathbf{O}$ form a categorical adjunction, expressed by the natural isomorphism of the Hom-bifunctors:

$$
\Psi: \mathbf{H o m}_{\text {Sets }^{2 t}}(\overrightarrow{\mathbf{A}}(-),-) \cong \mathbf{H o m}_{\text {Sets }}(-, \overline{\mathbf{A}}(-))
$$

Equivalently, $\overrightarrow{\mathbf{A}}, \overleftarrow{\mathbf{A}}$ have mutual functorial gauges with respect to each other if and only if the bifunctor $\wedge(-,-):$ Sets $\times$ Sets $^{\mathfrak{A}} \rightarrow$ Sets is birepresentable, that is, we have a series of natural isomorphisms of bifunctors:

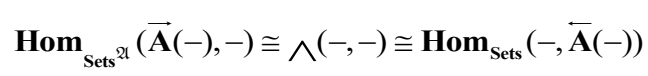

If we consider a signal transmitter/receiver $\chi \in \wedge(\mathcal{K}, \mathbf{P})$, then we realize that $\chi$ is actually a cone. Hence, the corresponding morphisms, induced by the birepresentability of the bifunctor $\wedge(-,-)$ within Sets, Sets $^{\mathfrak{A}}$ respectively, that is:

$$
\begin{aligned}
& g(\chi): \mathcal{K} \rightarrow \overline{\mathbf{A}}(\mathbf{P}) \\
& h(\chi): \overrightarrow{\mathbf{A}}(\mathcal{K}) \rightarrow \mathbf{P}
\end{aligned}
$$

are conjugates with respect to the cone signal transmitter/receiver $\chi$. The morphism $h(\chi): \Delta(\mathcal{K}) \rightarrow \mathbf{P}$ is a natural transformation in Sets $^{\mathfrak{A}}$ from the constant $\mathcal{K}$-valued copresheaf $\Delta(\mathcal{K}): \mathfrak{A} \rightarrow$ Sets to the copresheaf $\mathbf{P}: \mathfrak{A} \rightarrow$ Sets . The conjugate morphism of $h(\chi)$ with 
respect to the cone $\chi$, that is, $g(\chi): \mathcal{K} \rightarrow \overline{\mathbf{A}}(\mathbf{P})$ is a morphism in Sets (representing the cone $\chi$ ) from $\mathcal{K}$ to the set $\overline{\mathbf{A}}(\mathbf{P})=\mathbf{O}(\mathbf{P})$ obtained by the application of the sought right adjoint $\mathbf{O}$ on a copresheaf $\mathbf{P}$. By further taking into account that the functor $\mathbf{O}$ (as a right adjoint to $\Delta$ ) should assign to each $\mathbf{P}$ in Sets $^{\mathfrak{A}}$ an object $\mathbf{O}(\mathbf{P})$ in Sets representing the functor:

$$
\operatorname{Hom}_{\text {Sets }^{\mathfrak{A}}}(\Delta(-), \mathbf{P})=\mathbf{y}^{\mathrm{P}} \circ \Delta: \text { Sets } \rightarrow \text { Sets }
$$

or equivalently that the pair $\left(\mathbf{O}(\mathbf{P}), \varepsilon_{\mathrm{P}}\right)$ should be a universal gauge for the functor $\mathbf{y}^{\mathrm{P}} \circ \Delta$, we conclude that:

$$
\mathbf{O}(\mathbf{P})=\overline{\mathbf{A}}(\mathbf{P})=\operatorname{Lim}(\mathbf{P})
$$

where

$$
\mathbf{O}=\overline{\mathbf{A}}=\operatorname{Lim}: \text { Sets }^{\mathfrak{A}} \rightarrow \text { Sets }
$$

is the sought right adjoint to $\Delta$. Actually, the identification of the right adjoint to $\Delta$ with the functor $\operatorname{Lim}:$ Sets $^{\mathfrak{A}} \rightarrow$ Sets is immediate from the identification of a signal transmitter/receiver $\chi \in \wedge(\mathcal{K}, \mathbf{P})$ with a cone and the requirement of universality of the gauge formed by $\operatorname{Lim}(\mathbf{P})$ for each $\mathbf{P}$ in $\operatorname{Sets}^{\mathfrak{A}}$.

Dually, if we consider the diagonal functor $\Delta$ as a functor of the form $\mathbf{G}$, then it has a functorial gauge if and only if it has a left adjoint functor of the form $\mathbf{F}$, denoted by:

$$
\Lambda: \text { Sets }^{\mathfrak{A}} \rightarrow \text { Sets }
$$

Moreover, the oppositely pointing functors $\Delta, \mathbf{O}$ have mutual functorial gauges with respect to each other if and only if $\Delta$ is right adjoint to $\Lambda$ and $\Lambda$ is left adjoint to $\Delta$, such that the functors $\overrightarrow{\mathbf{A}}:=\Lambda, \overline{\mathbf{A}}:=\Delta$ form a categorical adjunction, expressed by the natural isomorphism of the Hom-bifunctors:

$$
\Xi: \mathbf{H o m}_{\text {Sets }}(\overrightarrow{\mathbf{A}}(-),-) \cong \mathbf{H o m}_{\text {Sets }^{24}}(-, \overline{\mathbf{A}}(-))
$$


Equivalently, $\quad \overrightarrow{\mathbf{A}}=\boldsymbol{\Lambda}, \quad \overline{\mathbf{A}}=\Delta$ have mutual functorial gauges with respect to each other if and only if the bifunctor $\wedge^{(-,-)}$: Sets $^{\mathfrak{A}} \times$ Sets $\rightarrow$ Sets is birepresentable, that is, we have a series of natural isomorphisms of bifunctors:

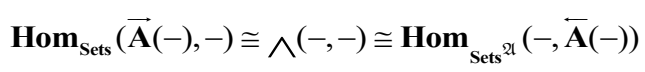

If we consider a signal transmitter/receiver $\psi \in \wedge(\mathbf{P}, \mathcal{K})$, then we realize that $\psi$ is actually a cocone. Hence, the corresponding morphisms, induced by the birepresentability of the bifunctor $\wedge^{(-,-)}$ within Sets $^{\mathfrak{A}}$, Sets, respectively, that is:

$$
\begin{aligned}
& f(\psi): \mathbf{P} \rightarrow \overline{\mathbf{A}}(\mathcal{K}) \\
& x(\psi): \overrightarrow{\mathbf{A}}(\mathbf{P}) \rightarrow \mathcal{K}
\end{aligned}
$$

are conjugates with respect to the cocone signal transmitter/receiver $\psi$. The morphism $f(\psi): \mathbf{P} \rightarrow \overleftarrow{\mathbf{A}}(\mathcal{K})$ is a natural transformation in Sets $^{\mathfrak{A}}$ from the copresheaf $\mathbf{P}: \mathfrak{A} \rightarrow$ Sets to the constant $\mathcal{K}$-valued copresheaf $\Delta(\mathcal{K}): \mathfrak{A} \rightarrow$ Sets. The conjugate morphism of $f(\psi)$ with respect to the cocone $\psi$, viz. $x(\psi): \overrightarrow{\mathbf{A}}(\mathbf{P}) \rightarrow \mathcal{K}$ is a morphism in Sets (representing the cocone $\psi$ ) from the set $\overrightarrow{\mathbf{A}}(\mathbf{P})=\Lambda(\mathbf{P})$ obtained by the application of the sought left adjoint $\Lambda$ on a copresheaf $\mathbf{P}$ to a set $\mathcal{K}$. By further taking into account that the functor $\Lambda$ (as a left adjoint to $\Delta)$ should assign to each $P$ in Sets $^{\mathfrak{A}}$ an object $\Lambda(\mathbf{P})$ in Sets representing the functor:

$$
\operatorname{Hom}_{\text {Sets }^{\mathfrak{A}}}(\mathbf{P}, \Delta(-))=\mathbf{y}_{\mathbf{P}} \circ \Delta: \text { Sets } \rightarrow \text { Sets }
$$

or equivalently, that the pair $\left(\Lambda(\mathbf{P}), \eta_{\mathrm{P}}\right)$ should be a universal gauge for the functor $\mathbf{y}_{\mathrm{P}} \circ \Delta$ we conclude that:

$$
\Lambda(\mathbf{P})=\overrightarrow{\mathbf{A}}(\mathbf{P})=\operatorname{Colim}(\mathbf{P})
$$

where

$$
\mathbf{\Lambda}=\overrightarrow{\mathbf{A}}=\text { Colim }: \text { Sets }^{\mathfrak{A}} \rightarrow \text { Sets }
$$


is the sought left adjoint to $\Delta$. Again, the identification of the left adjoint to $\Delta$ with the functor Colim: Sets $^{\mathfrak{A}} \rightarrow$ Sets follows immediately from the identification of a signal transmitter/receiver $\psi \in \wedge(\mathbf{P}, \mathcal{K})$ with a cocone and the requirement of universality of the gauge formed by $\operatorname{Colim}(\mathbf{P})$ for each $\mathbf{P}$ in $\mathbf{S e t s}^{\mathfrak{A}}$.

We conclude that the functors Lim:Sets ${ }^{\mathfrak{A}} \rightarrow$ Sets , Colim $:$ Sets $^{\mathfrak{A}} \rightarrow$ Sets are right and left adjoints correspondingly of the diagonal functor $\Delta$. More precisely:

i The functors $\Delta:$ Sets $\rightarrow$ Sets $^{\mathfrak{A}}, \quad$ Lim $:$ Sets $^{\mathfrak{A}} \rightarrow$ Sets have mutual functorial gauges with respect to each other forming a categorical adjunction, induced by the birepresentability of the bifunctor of cones $\wedge^{(-,-)}:$Sets $\times$Sets $^{\mathfrak{A}} \rightarrow$ Sets , within Sets , Sets $^{\mathfrak{A}}$ respectively:

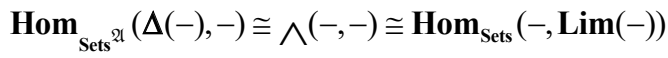

ii The functors Colim $:$ Sets $^{\mathfrak{A}} \rightarrow$ Sets $, \quad \Delta:$ Sets $\rightarrow$ Sets $^{\mathfrak{A}}$ have mutual functorial gauges with respect to each other forming a categorical adjunction, induced by the birepresentability of the bifunctor of cocones $\wedge^{(-,-)}:$Sets $^{\mathfrak{A}} \times$ Sets $\rightarrow$ Sets, within Sets $^{\mathfrak{A}}$, Sets respectively:

$$
\operatorname{Hom}_{\text {Sets }}(\operatorname{Colim}(-),-) \cong \wedge(-,-) \cong \operatorname{Hom}_{\text {Sets }^{\mathfrak{A}}}(-, \Delta(-))
$$

Furthermore, we can show immediately that right adjoint functors commute with limits, while left adjoint functors commute with colimits. It is instructive to think of these properties of left/right adjoint functors as preservation properties induced by the requirement that functorial gauges should apply. Obviously, these properties can be used conversely in order to prove that a given functor does not have a functorial gauge by showing that it does not preserve limits or colimits. Finally, the above arguments remain valid if we replace the category of Sets by any other complete category of algebras for the case [i], and by any other cocomplete category of algebras for the case [ii] respectively.

Along the same lines, we can also show that for any small category $\mathfrak{A}$, every presheaf $\mathbf{P}$ in the functor category of presheaves $\operatorname{Sets}^{\mathfrak{A}^{o p}}$, is a colimit of standard (representable) functors, by which we mean that it 
constitutes a colimit of functorial gauges. This is expressed as follows: We consider the category of elements of a presheaf $\mathbf{P}$ in $\operatorname{Sets}^{\mathfrak{A l}^{o p}}$, that is the category $\int(\mathbf{P}, \mathfrak{A})$. Projection on the second coordinate of $\int(\mathbf{P}, \mathfrak{A})$, gives a functor:

$$
\int_{\mathbf{P}}: \int(\mathbf{P}, \mathfrak{A}) \rightarrow \mathfrak{A}
$$

defining the split discrete fibration induced by $\mathrm{P}$, where $\mathfrak{A}$ is the base indexing or localizing category of the fibration. Then, as a consequence of the Yoneda lemma we obtain a natural transformation:

$$
\checkmark: \operatorname{Colim}\left(\mathbf{y} \circ \int_{P}\right) \cong \mathbf{P}
$$

which is a natural isomorphism. More precisely, we may consider a signal transmitter/receiver from $\left(\mathbf{y} \circ \int_{\mathbf{P}}\right)$ to $\mathbf{P}$, or equivalently from $\operatorname{Hom}\left(\int_{\mathbf{P}}(-),-\right)$ to $\mathbf{P}$. This is actually a cocone, which for every $(p, \mathcal{A})$ in $\int(\mathbf{P}, \mathfrak{A})$, provides a component $\mathbf{y}_{\mathcal{A}}$, and thus, becomes representable within $\operatorname{Sets}^{\mathfrak{A l}^{\text {op }}}$ in terms of the natural transformation $\mathbf{y}_{\mathcal{A}} \rightarrow \mathbf{P}$. Now, by the Yoneda lemma we have a bijective correspondence between natural transformation $\mathbf{y}_{\mathcal{A}} \rightarrow \mathbf{P}$ and elements $p$ of $\mathbf{P}(\mathcal{A})$. Thus, each arrow in the cocone of the form $\mathbf{y}_{\mathcal{A}} \rightarrow \mathbf{P}$ for each $\mathcal{A}$ in $\mathfrak{A}$ is a corresponding element $p$ of $\mathbf{P}(\mathcal{A})$. It is straightforward to see that this cocone in $\operatorname{Sets}^{\mathfrak{A l}^{o p}}$ is a universal one, and thus the natural transformation $\operatorname{Colim}\left(\mathbf{y} \circ \int_{\mathbf{P}}\right) \rightarrow \mathbf{P}$ is a natural isomorphism.

Another important consequence of the existence of mutual functorial gauges for two oppositely pointing functors between the same categories is the following: We have seen that $\overrightarrow{\mathbf{A}}: \mathfrak{C}^{o p} \rightarrow \mathfrak{L}, \overline{\mathbf{A}}: \mathfrak{L} \rightarrow \mathfrak{C}^{o p}$ have mutual functorial gauges with respect to each other if and only if there exist a natural transformation of the identity functor on $\mathfrak{C}^{o p}$ (unit natural transformation) $\eta: \mathbf{i d}_{\mathfrak{C}^{\text {op }}} \rightarrow \overrightarrow{\mathbf{A}} \overrightarrow{\mathbf{A}}$, as well as a natural transformation of the identity functor on $\mathfrak{L}$ (counit natural transformation) $\quad \varepsilon: \overrightarrow{\mathbf{A}} \overleftarrow{\mathbf{A}} \rightarrow \mathbf{i d}_{\mathfrak{L}}$ obeying the triangular identities: $i d_{\overline{\mathbf{A}}}=\varepsilon \overrightarrow{\mathbf{A}} \circ \overrightarrow{\mathbf{A}} \eta, i d_{\overline{\mathbf{A}}}=\overleftarrow{\mathbf{A}} \varepsilon \circ \eta \overleftarrow{\mathbf{A}}$. If both the unit and the counit natural transformations of the adjunction formed by the functors $\overrightarrow{\mathbf{A}}, \overline{\mathbf{A}}$, are 
natural isomorphisms then we obtain an equivalence of the categories $\mathfrak{C}^{o p}, \mathfrak{L}$, that is, we obtain a functorial duality:

$$
\mathfrak{C}^{o p} \simeq \mathfrak{L}
$$

Thus, the notion of functorial duality is a consequence of the existence of mutual functorial gauges for two oppositely pointing functors between the same categories when both the unit and counit natural transformations of the formed adjunction are natural isomorphisms.

We reached the conclusion that two oppositely pointing functors between the same categories, $\overrightarrow{\mathbf{A}}: \mathfrak{C}^{o p} \rightarrow \mathfrak{L}$ and $\overline{\mathbf{A}}: \mathfrak{L} \rightarrow \mathfrak{C}^{o p}$ form a categorical adjunction, induced by the requirement of birepresentability of the bifunctor $\wedge^{(-,-)}$, where $\overrightarrow{\mathbf{A}}$ is the left adjoint functor of the adjunction and symmetrically $\overline{\mathbf{A}}$ is the right adjoint functor of the adjunction, or equivalently $\overrightarrow{\mathbf{A}}$ and $\overline{\mathbf{A}}$ have mutual functorial gauges with respect to each other, if and only if there exist a natural transformation of the identity functor on $\mathfrak{C}^{o p}$ (unit natural transformation):

$$
\eta: \mathbf{i d}_{\mathfrak{c}^{\text {op }}} \rightarrow \overleftrightarrow{\mathbf{A}} \overrightarrow{\mathbf{A}}
$$

as well as a natural transformation of the identity functor on $\mathfrak{L}$ (counit natural transformation):

$$
\varepsilon: \overrightarrow{\mathbf{A}} \overleftarrow{\mathbf{A}} \rightarrow \mathbf{i d}_{\mathfrak{L}}
$$

obeying the following identities:

$$
\begin{aligned}
& i d_{\overline{\mathbf{A}}}=\varepsilon \overrightarrow{\mathbf{A}} \circ \overrightarrow{\mathbf{A}} \eta \\
& i d_{\overline{\mathbf{A}}}=\overline{\mathbf{A}} \varepsilon \circ \eta \overline{\mathbf{A}}
\end{aligned}
$$

The composite endofunctor $\mathbf{T}:=\overrightarrow{\mathbf{A}} \overrightarrow{\mathbf{A}}: \mathfrak{C}^{o p} \rightarrow \mathfrak{C}^{o p}$, together with the natural transformations $\omega: \mathbf{T} \circ \mathbf{T} \rightarrow \mathbf{T}$, called multiplication, and also, $\eta: \mathbf{i d}_{\mathfrak{C}^{\text {op }}} \rightarrow \mathbf{T}$, called unit, where $\mathbf{i d}_{\mathfrak{C}^{\text {op }}}$ is the identity functor on $\mathfrak{C}^{o p}$, is defined as a monad $(\mathbf{T}, \omega, \eta)$ on the category $\mathfrak{C}^{o p}$, provided that, the diagrams below commute for each object $\mathcal{C}$ of $\mathfrak{C}^{o p}$; 

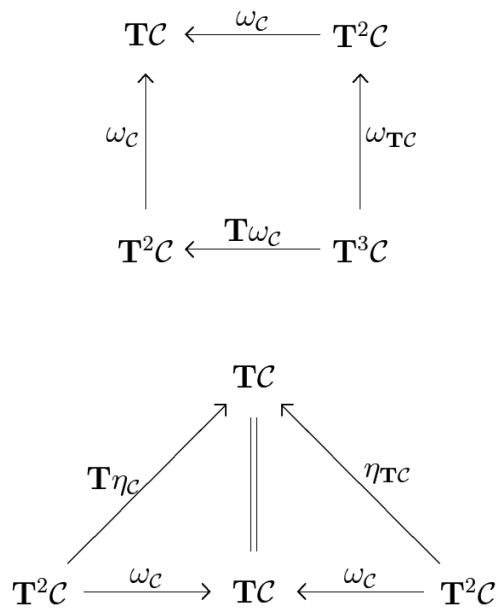

From the above, we conclude that a monad $(\mathbf{T}, \omega, \eta)$ on the category $\mathfrak{C}^{o p}$ can be understood as a monoid in the category of endofunctors of $\mathfrak{C}^{o p}$ with the morphisms being the natural transformations between them.

We have another dual relation; the composite endofunctor $\mathbf{O}:=\overrightarrow{\mathbf{A}} \overline{\mathbf{A}}: \mathfrak{L} \rightarrow \mathfrak{L}$, together with the natural transformations $\delta: \mathbf{O} \rightarrow \mathbf{O} \circ \mathbf{O}$, called comultiplication, and also, $\varepsilon: \mathbf{O} \rightarrow \mathbf{i d}_{\mathfrak{L}}$, called counit, where $\mathbf{i d}_{\mathfrak{L}}$ is the identity functor on $\mathfrak{L}$, is defined as a comonad $(\mathbf{O}, \delta, \varepsilon)$ on the category $\mathfrak{L}$, provided that the diagrams below commute for each object $\mathcal{L}$ of $\mathfrak{L}$;

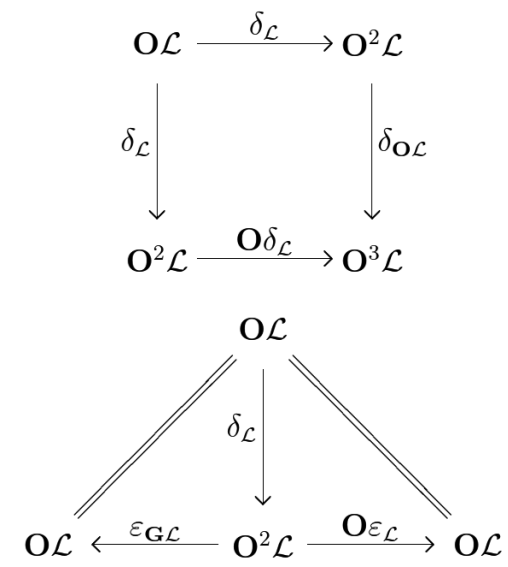


For a comonad $(\mathbf{O}, \delta, \varepsilon)$ on $\mathfrak{L}$, a $\mathbf{O}$-coalgebra (comodule) is an object $\mathcal{L}$ of $\mathfrak{L}$, being equipped with a structural map $\kappa: \mathcal{L} \rightarrow \mathbf{O} \mathcal{L}$, such that the following conditions are satisfied:

$$
\begin{gathered}
1_{\mathcal{L}}=\varepsilon_{\mathcal{L}} \circ \kappa: \mathcal{L} \rightarrow \mathcal{L} \\
\mathbf{O} \kappa \circ \kappa=\delta_{\mathcal{L}} \circ \kappa: \mathcal{L} \rightarrow \mathbf{O}^{2} \mathcal{L}
\end{gathered}
$$

With the above obvious notion of morphism, this gives a category $\mathfrak{L}_{\mathbf{O}}$ of all $\mathbf{O}$-coalgebras.

By dual correlation, if $(\mathbf{T}, \omega, \eta)$ is a monad on the category $\mathfrak{C}^{o p}$, we define the category of $\mathfrak{C}^{o p}{ }_{\mathbf{T}}$-algebras (modules) as follows: Its objects are pairs $\left(\mathcal{C}, \mu_{\mathcal{C}}\right)$, where, $\mathcal{C}$ in $\mathfrak{C}^{o p}$, and, $\mu: \mathbf{T}(\mathcal{C}) \rightarrow \mathcal{C}$ is a morphism in $\mathfrak{C}^{o p}$, such that, the following conditions are satisfied:

$$
\begin{gathered}
1_{\mathcal{C}}=\mu \circ \eta_{\mathcal{C}}: \mathcal{C} \rightarrow \mathcal{C} \\
\mu \circ \mathbf{T} \mu=\mu \circ \omega_{\mathcal{C}}: \mathbf{T}^{2} \mathcal{C} \rightarrow \mathcal{C}
\end{gathered}
$$

Since an adjunction between two categories $\mathfrak{C}^{o p}, \mathfrak{L}$, defined by a pair of adjoint functors $\overrightarrow{\mathbf{A}}: \mathfrak{C}^{o p} \rightarrow \mathfrak{L}$ and $\overline{\mathbf{A}}: \mathfrak{L} \rightarrow \mathfrak{C}^{o p}$ always gives rise to a monad on the category $\mathfrak{C}^{o p}$, viz. $(\mathbf{T}=\overleftarrow{\mathbf{A}} \overrightarrow{\mathbf{A}}, \omega=\overleftarrow{\mathbf{A}} \varepsilon \overrightarrow{\mathbf{A}}, \eta)$, as well as to a comonad on the category $\mathfrak{L}$, viz. $(\mathbf{O}=\overrightarrow{\mathbf{A}} \overleftarrow{\mathbf{A}}, \delta=\overrightarrow{\mathbf{A}} \eta \overleftarrow{\mathbf{A}}, \varepsilon)$, we say that $\overrightarrow{\mathbf{A}}$ and $\overline{\mathbf{A}}$ have mutual functorial gauges with respect to each other, if and only if a monad-comonad pair exists as above. 

